\title{
Pre-acid system for improving the hydraulic fracturing effect in low-permeability tight gas reservoir
}

\author{
Nianyin $\mathrm{Li}^{1}$ (D) · Fei Chen ${ }^{2} \cdot$ Jiajie $\mathrm{Yu}^{1} \cdot$ Peihong Han ${ }^{1} \cdot$ Jia Kang $^{1}$
}

Received: 30 October 2020 / Accepted: 27 February 2021 / Published online: 17 March 2021

(c) The Author(s) 2021

\begin{abstract}
Hydraulic fracturing is an important technical means to improve the development effect of low-permeability oil and gas reservoirs. However, for low pressure, low-permeability, tight, and high-clay sandstone gas reservoirs, conventional propped fracturing can cause serious damage to the reservoir and restrict the fracturing effect. The pre-acid fracturing technology combines acid treatment technology with sand-fracturing technology. A pre-acid system that meets special performance requirements is injected before fracturing. The pre-acid reduces the formation fracture pressure and removes clay damage. During acid flowback, the fracturing fluid is promoted to break the gel, dissolve the fracturing fluid residue and polymer filter cake, clean the supporting cracks, and effectively improve the fracturing effect. This study analyzes the process principle and technical advantages of the pre-acid fracturing technology based on the laboratory evaluation of the fracturing damage mechanism of low-permeability tight gas reservoirs. To meet the performance requirements of low-permeability tight gas reservoirs and pre-acid fracturing technology, a set of polyhydrogen acid system with long-lasting slow reactivity, low damage, and low corrosion was developed and used as the pre-fracturing acid. The acid system is mainly composed of the main agent SA601 and the auxiliary agent SA701. Then, on the basis of laboratory experiments, this acid system is used as the fracturing pre-acid to evaluate the fracturing improvement effect. The results show that the fracturing fluid system can better dissolve the fracturing fluid filter cake and remove the fracturing fluid damage.
\end{abstract}

Keywords Hydraulic fracturing $\cdot$ Pre-acid $\cdot$ Tight gas reservoir $\cdot$ Acidification $\cdot$ Reservoir damage

\section{Introduction}

For a long time, hydraulic propped fracturing has become an important technical measure for increasing the production of low porosity and permeability oil and gas reservoirs (Holditch 1979; Gdanski and Fulton 2006). However, conventional propped fracturing still has a series of problems, which restrict the fracturing effect. The effect of hydraulic fracturing is severely restricted, mainly due to a series of problems that cannot be ignored in conventional propped fracturing (Volk and Gall 1983; Xu et al 2011; Kim and Losacano 1985; Wang

Nianyin Li

lnyswpu@163.com

1 State Key Lab of Oil and Gas Reservoir Geology and Exploitation, Southwest Petroleum University, Chengdu 610500, Sichuan, China

2 Changqing Downhole Technical Service Company of Chuanqing Drilling Engineering Co., Ltd., CNPC, Xian, Shanxi, China et al. 2008; Cheng et al 2018; Tan et al. 2017). For tight gas reservoirs with low pressure and permeability, its damage has a more significant impact on the effect of fracturing stimulation. Mainly reflected in: (1)The formation rock is tight, resulting in the high fracture pressure The formation rock is tight, resulting in the high fracture pressure of the reservoir rock, which is reflected in the high wellhead pressure in the sandfracturing construction, and it is difficult to open the reservoir under the pressure of the existing wellhead device; (2Pressure Fracture fluid residues remaining in supporting fractures or natural fractures and reservoir pores block the seepage channel and damage the seepage channel; (3)The fracturing fluid leaks into the reservoir and causes damage to the reservoir; (4) The proppant is broken and the rock particles in the reservoir cause the supporting fracture (5)The damage caused by the fracturing fluid filter cake to the fracture wall; @ 6 Hydraulic fracturing cannot effectively remove inorganic scaling and the damage to the reservoir caused by solid substances entering the reservoir during drilling or completion. However, acidification (acid fracturing) has advantages in reducing formation 
fracture and fracturing, and releasing damage from drilling and completion and fracturing (Guo et al 2017; Hou et al 2019; Li et al 2012). To further improve the fracturing effect and fracturing efficiency, it is necessary to redefine the idea of deep reconstruction of low-permeability tight gas reservoirs, integrate the advantages of acidification into the fracturing process, and consider the acidification in high fracture pressure, tight sandstone reservoirs, and sensitive sandstones. The role of reservoir reformation is to improve the fracturing effect. Consequently, the pre-acid fracturing technology is proposed (Wu and Sharma 2016; Tang 2017). This technology injects a pre-acid system with special performance requirements into the formation before conventional hydraulic propped fracturing, and then enters the conventional hydraulic sand-fracturing process (Gensheng et al 2012). Compared with conventional hydraulic propped fracturing, the most significant advantages of the pre-acid fracturing technology include: First, the pre-acid pretreatment of the reservoir during the pre-acid injection process reduces the formation fracture pressure; The sensitive minerals of the formation can improve the permeability of the formation near the fractures of the reservoir, prevent the expansion and dispersion of the clay and prepare for the subsequent injection of the fracturing sand-carrying fluid (Peng et al. 2013). Second, when fluid flows back into the well, fracturing fluid flows back first, followed by residual acid. It can make full use of the degradation and cleaning effects of low $\mathrm{pH}$ residual acid, promote the gel breaking of fracturing fluid, dissolve the fracturing fluid filter cake, clean support fractures and improve the conductivity of support fractures (Jiang et al 2015). This process combines acid treatment technology with sand-fracturing technology, which can not only play the advantages of conventional fracturing to reform the reservoir, but also significantly solve its existing problems and improve the fracturing effect (Qasem et al. 2007). The optimization of the pre-acid acid solution system is different from the previous acidification acid fracturing because the pre-acid sanding-fracturing process not only considers the factors of the reaction degree of the acid and the rock but also considers factors such as late fracturing and drainage (Dang et al 2019). An ideal pre-acid fracturing acid system must not only have a certain dissolution capacity for reservoir minerals but also have the characteristics of longer action time and low damage to the proppant, so that it can promote pressure in the flowback of the acid. The fracturing fluid breaks the gel, dissolves the fracturing fluid filter cake, and cleans the supporting fractures to improve the fracturing effect. Based on the characteristics of low-permeability tight gas reservoirs and the performance requirements of the preacid fracturing process for the pre-acid system, a new type of chelating acid system - polyhydroacid is developed as the pre-acid. The performance of the pre-acid solution was systematically evaluated in terms of reaction performance, corrosion ability, and acidification effect. Also, the mechanism of the pre-acid in improving the fracturing effect was evaluated in terms of the pre-acid reducing rock fracture pressure, promoting fracturing fluid gel breaking, reducing clay damage, cleaning the matrix, and supporting cracks.

\section{Methodology}

Firstly, this paper analyzes the damage mechanism of hydraulic fracturing in low permeability tight gas reservoir through the experiments of dynamic adsorption and retention of fracturing fluid and core damage caused by fracturing fluid filtrate; then, through the experiments of solution rate test, retaining performance test, inhibition of secondary precision test, solution rate of proppant by pre-acid test, corrosion performance of pre-acid system test, and minimum energy spectrum analysis after pre-acid reactions with rock, the suitable pre-acid formula for low permeability tight gas reservoir is selected; finally, through the experiments of reducing rock strength by pre-acid damage, the influence of pre-acid on gel breaking of fracturing fluid, the influence of pre-acid on clay expansion and particle migration, the cleaning of matrix pores and supporting fractures by preacid, the effect of improving fracturing under the pre-acid formula is evaluated.

\section{Damage of hydraulic fracturing in low-permeability tight gas reservoirs}

In the process of drilling and completion, the use of inappropriate drilling fluid damages the oil layer, and also causes the oil and gas layer to lose its due production capacity. This has been a long concern; however, various measures have been proposed to prevent the drilling and completion fluid from affecting the formation (Zhang et al 2010; Hajiabadi et al. 2020). Also, in the process of hydraulic fracturing, the jellyfracturing fluid used will also cause damage to the oil and gas reservoirs similar to the process of drilling and completion (Wang et al 2020). The damage of hydraulic fracturing to productivity is also different from the process of drilling and completion. In terms of nature, it is shown as follows: (1) Because a large amount of breaker is lost to the oil layer through the filter cake and the filtrate, it greatly reduces its impact on the filter cake. Glue breaking and hydration, thereby increasing the retention time of the filter cake on the fracture wall, affecting the smooth entry of oil and gas from the oil layer into the fracture; (2) After the fracture is closed, the residue concentration in the sand-filled fracture greatly exceeds the concentration during surface injection. Thus, greatly reducing the conductivity of the sand-filled cracks; (3) when the discharge intensity exceeds the requirement, the fracturing fluid residues and the particles in the fracture will move, and they may get stuck in certain parts of the 
fracture, causing further damage to the fracture conductivity. (4) After the fracturing filtrate invades the reservoir during the fracturing construction process, due to the retention of the capillary force, the formation driving pressure cannot completely drain the filtrate out of the formation. The water saturation of the reservoir will increase and the oil gas permeability will decrease. For low-permeability and ultra-lowpermeability reservoirs, water blocking is more serious. During the fracturing process, the deeper the penetration depth of the fracturing fluid, the more difficult it is to discharge the retained water. For tight reservoirs, due to low porosity, water blocking damage is more serious. In the low pressure and tight reservoirs, once the reservoir is water-locked, it is very difficult to remove the water lock damage.

The damage of fracturing fluid in the oil layer is more intense than the drilling fluid (Huang et al 2020; Fu et al 2020; Hou et al. 2019; Lufeng et al. 2019), which is specifically expressed as (1) The fluid loss area of fracturing fluid is larger than that of opening the oil layer; (2) fracture rupture and extension in the process, the pressure difference between inside and outside the fracture is much larger than the pressure difference between the bottom of the well and the oil layer when the oil layer is drilled. Therefore, under the same conditions, the damage of fracturing fluid in the production capacity of the oil layer should not be taken lightly.

Holditch (1979) showed that capillary force and relative permeability damage are important factors affecting the flowback of fractured wells. Gdanski and Fulton (2006) illustrated Holditch's view using numerical simulation; Volk and Gall (1983) established an evaluation of the fracture wall bedrock. According to the empirical method of damage, the damage caused by the fluid loss of fracturing fluid should not be ignored; Xu et al (2011) experimentally studied the formation process of the guar gum fracturing fluid filter cake and found that the thickness of the filter cake is linearly related to the fluid loss. The filter cake has a yield stress, which determines its removability. Kim and Losacano (1985) studied the damage of cross-linking agent residues to proppant conductivity. They believed that the damage degree depends on the fracturing fluid, but it is not directly proportional to the polymer concentration used; Wang and Holditch (2008) used computer simulation to study the relationship between fracturing fluid residue cleaning in tight gas reservoirs and natural gas recovery;

\section{Evaluation of fracturing fluid dynamic adsorption and retention damage}

Different concentrations of hydroxypropyl guanidine solution were prepared, and use proppant dynamic adsorption retention damage experimental device to conduct experiments (Fig. 1).

\section{The microscopic morphology of fracturing fluid adsorption on the surface of proppant}

We used clean water and hydroxypropyl guar gum solution to drive the ceramsite proppant belt for $2 \mathrm{~h}$, then place it in a constant temperature blast drying oven and observe the adsorption and retention of the hydroxypropyl guar gum inside the proppant belt. Finally, observe the proppant before and after the injury through an electron microscope.
Fig. 1 Experimental device for proppant dynamic adsorption and retention damage

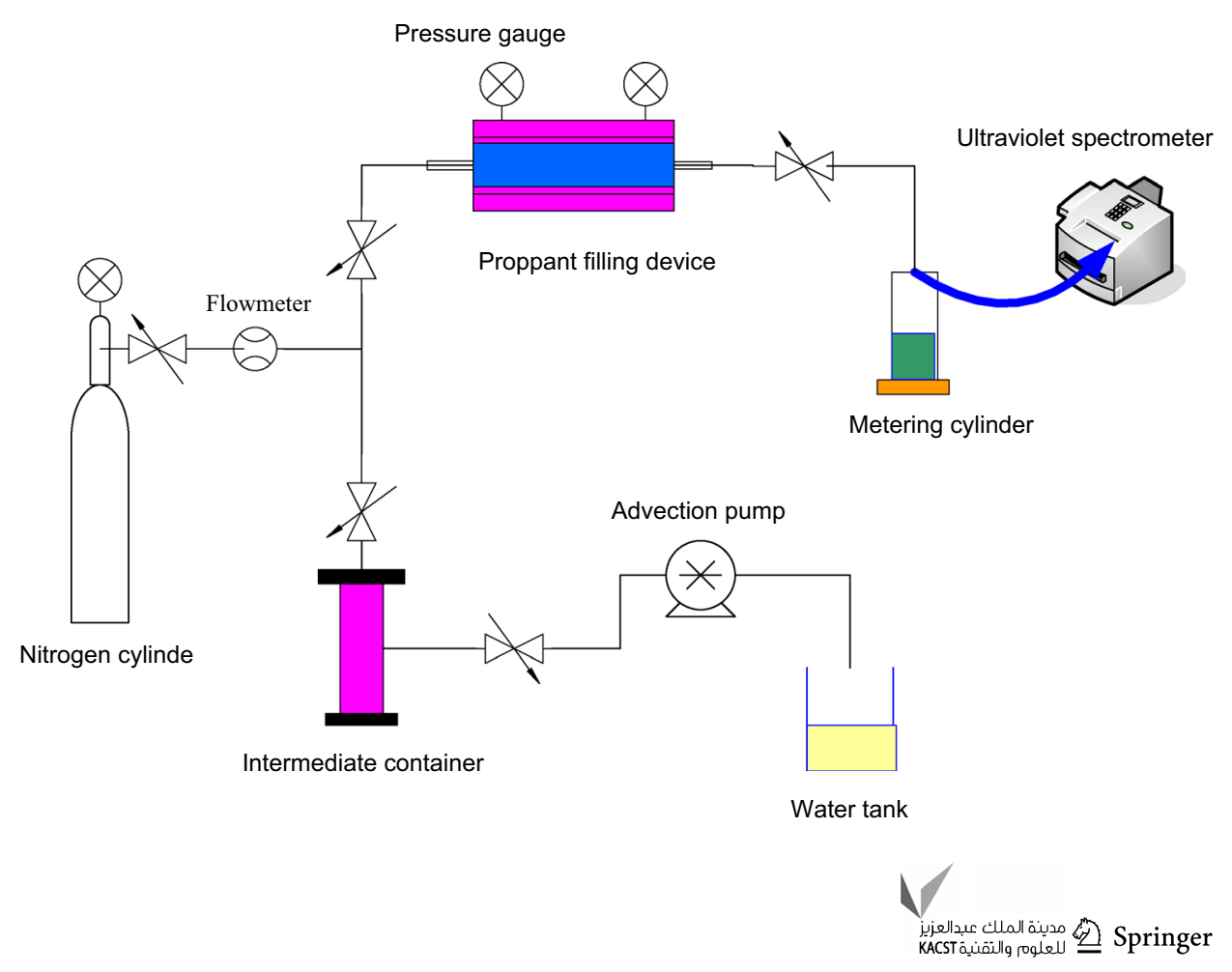




\section{Experimental evaluation of damage of fracturing fluid filtrate to core matrix and micro-fractures}

The experiment process is as follows:

1. Determine the core permeability $\mathrm{K}_{1}$ before damage: the flow medium ( $4 \% \mathrm{NH}_{4} \mathrm{Cl}$ as simulated formation water) is squeezed into the core from the reverse end of the core holder for displacement, and the flow rate of the flow medium is lower than the critical flow rate. Until the flow rate and pressure difference are stable, the stabilization time is not less than $60 \mathrm{~min}$;

2. Use the high temperature and high-pressure flow meter to forward the pressure fluid and the gel breaker into the core, and the displacement volume is $10 \mathrm{PV}$; the determination of core permeability $K_{2}$ after damage;

3. Measure the permeability $K_{2}$ of the flowing medium after the core is damaged by fracturing fluid filtrate;

4. The matrix permeability damage rate is calculated by

$$
\eta_{\mathrm{d}}=\frac{K_{1}-K_{2}}{K_{1}} \times 100 \%
$$

\section{Design experiment of pre-acid system}

The mechanism of pre-acid propped fracturing is shown in Fig. 2. At a pressure higher than the fracture pressure, the pre-acid fracturing technology pumps acid into the formation so that a part of the acid is at the forefront of the fracture, whereas a part is filtered to both sides of the fracture wall, and then a certain isolation fluid is squeezed into the fracturing. Liquid phase isolation prevents the fracturing fluid from hydrating and breaking gel after encountering acid, which will cause fracturing failure. Then, the sand-carrying fluid and displacement fluid is continuously pumped. After the fracture is completed, the fracturing fluid and acid will drain back. Relying on the injection of pre-acid to relieve

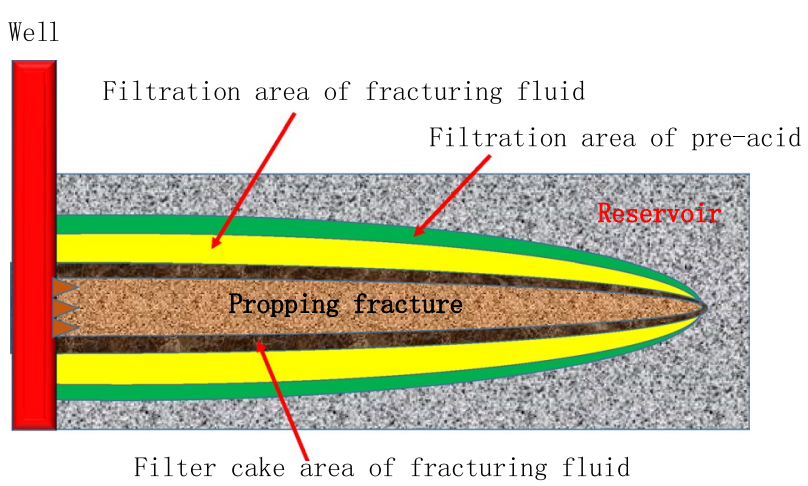

Fig. 2 Schematic diagram of the action mechanism of pre-acid propped fracture clay damage, drilling and completion damage, reduce formation fracture pressure, in the process of fluid drainage after fracturing, the long-acting pre-acid system promotes fracturing fluid gel breaking, cleaning matrix pores and supports fractures, in general, reduce the damage of fracturing fluid and improve the effect of increasing production.

Compared with conventional fracturing technology, the pre-acid fracturing technology has the following advantages: (1) Injecting acid before fracturing and pre-treating the formation with acid liquid, which can reduce the formation fracture pressure; (2) The clay stabilizer in the pre-acid and the dissolving effect of HF on clay minerals fundamentally reduces the damage caused by the clay expansion; (3) during fracturing, foreign fluid enters the formation and causes water lock, which makes it difficult to discharge fluid, which in turn affects the reforming effect. During pre-acid fracturing, replacing the fracturing fluid with acid can avoid damage to the reservoir caused by the fracturing fluid to a certain extent; (4) acid fluid can increase the degree of gel breaking of the fracturing fluid; acid flowback can regulate fracturing the $\mathrm{pH}$ value of the fluid is used to control the degree of cross-linking to realize the non-degradable gel breaking of the fracturing fluid; (5) the acid fluid can properly dissolve the fracturing fluid residue and filter cake, so as to achieve the role of cleaning and supporting cracks.

To ensure that the effect of the measures and performance requirements of the general acidification (acid fracturing) acid system, the pre-acid fracturing technology has special requirements for the pre-acid acid system. From the geological characteristics of the reservoir in the Eastern Sulige area, the analysis of potential damage factors in the process of drilling and completion, hydraulic fracturing, and the technical requirements of the pre-acid fracturing process, the performance characteristics of an optimal pre-acid system includes.

1. The pre-acid system has good compatibility with formation rocks and formation fluids;

2. The pre-acid system has a certain ability to dissolve reservoir minerals and can effectively remove various blockages;

3. The acid rock reaction speed is slow, and the effective action time of the acid is long, ensuring that the residual acid still has a strong reactivity in the formation after the injection of fracturing construction, so that the acid promotes fracturing during the flowback process the gel breaks the fluid, and at the same time, it dissolves the fracturing fluid filter cake and cleans the supporting cracks to improve the fracturing effect;

4. Generally, the construction time of propped fracturing is long, and secondary damage to the acid liquid is required to be small under the long-term action of acid in the formation; 
5. To ensure the strength of the proppant, the dissolution rate of the proppant by the pre-acid should be low.

Due to the characteristics of low-permeability tight reservoirs and the special performance requirements of the preacid fracturing process for the pre-acid, a set of long-lasting slow-rate and scale-inhibiting polyhydrogen acid system was developed. More so, Polyhydrogen acid is a new type of HF acid solution system. A special compound replaces $\mathrm{HCl}$ and fluoride salt to undergo hydrogenation reaction. Polyhydrogen acid is a moderately strong acid with ionization balance. The acid solution system can release multiple hydrogen ions through multistage ionization under different stoichiometric conditions. It is named "Multi-Hydrogen Acid" (Multi-Hydrogen Acid). The acid system is mainly composed of the main agent SA601 and the auxiliary agent SA701. Polyhydrogen acid is hydrogenated by a special compound instead of $\mathrm{HCl}$ and fluoride salt. Polyhydrogen acid is a strong acid with ionization balance. The acid solution system can release multiple hydrogen ions through a multistage ionization decomposition under different stoichiometric conditions.

Polyhydric acid controls the rate of HF generation through gradual ionization. In a low $\mathrm{pH}$ environment, the ionization of polyhydric acid is limited by the reaction equilibrium, so the concentration of hydrogen ions produced is relatively low. The buffer regulation system formed by polyhydrogen acid and fluoride salt, when HF reacts with rock minerals and is partly consumed, the balance is broken, and the reaction will proceed in the positive direction until the solution re-establishes a new balance. Therefore, as long as the acid concentration of the solution is large enough and the concentration of HF in the acid solution remains basically constant, the reaction between the acid solution and rock minerals will proceed at a relatively stable rate.

\section{Dissolution rate test}

A corrosion test was conducted on the reservoir core to understand the dissolution rate of the acid solution to the soluble matter in the core so as to initially determine the acid concentration. In addition to determining the acid concentration through the determination of dissolution, a comprehensive analysis was conducted based on the rock structure of the reservoir and the characteristics of the reservoir fluid.

Test temperature: $90^{\circ} \mathrm{C}$, dissolution time: $2 \mathrm{~h}$.

\section{Retarding performance test}

Since polyhydrogen acid is a HF acid solution system, it is impossible to directly measure the $\mathrm{pH}$ with a glass electrode, and an antimony electrode acidity transmitter is used to measure the electrode potential. The antimony electrode acidity transmitter is a $\mathrm{pH}$ measurement system composed of an antimony electrode and a reference electrode. In the tested acid solution, an antimony trioxide oxide layer will be formed on the surface of the antimony electrode, so a potential difference will be formed between the metal antimony surface and the antimony trioxide. The magnitude of the potential difference depends on the concentration of antimony trioxide, which corresponds to the moderate number of hydrogen ions in the measured acid solution. In the experiment, the electrode potential indirectly indicates the acidity of the polyhydrogen acid solution system.

\section{Inhibition of secondary precipitation test}

\section{(1) Suppresses metal ion precipitation}

According to the static scale inhibition test of polyhydrogen acid on $\mathrm{CaCO}_{3}$, the scale inhibition and dispersion performance of polyhydrogen acid is observed. (a: Polyhydrogen acid and alkali to adjust to neutral $+\mathrm{CaCl}_{2}+\mathrm{NaHCO}_{3}$, heating at $80{ }^{\circ} \mathrm{C}$ for $2 \mathrm{~h}$; b: polyhydrogen acid $+\mathrm{CaCl}_{2}+$ $\mathrm{NaHCO}_{3}$; c: $\mathrm{CaCl}_{2}+\mathrm{NaHCO}_{3}$, heating at $80{ }^{\circ} \mathrm{C}$ for $2 \mathrm{~h}$; d: $\mathrm{CaCl}_{2}+\mathrm{NaHCO}_{3}$ ).

(2) Inhibit silica gel precipitation

During the experiment, two solutions were prepared with $\mathrm{Na} 2 \mathrm{SiO} 3.9 \mathrm{H} 2 \mathrm{O}$ : solution one $\mathrm{Si}$ ion concentration was $4000 \mathrm{mg} / \mathrm{l}$, the solution contained $1.5 \mathrm{ml} \mathrm{SA} 601$ and $0.5 \mathrm{ml}$ SA701; solution two Si ion concentration was 2000 $\mathrm{mg} / \mathrm{l}$ without chelating agent for comparison experiment. The two solutions were both $50 \mathrm{ml}$ and the $\mathrm{pH}$ are about 2.5. Put the two solutions in a $95{ }^{\circ} \mathrm{C}$ constant temperature water bath to heat, cool, and filter. Use the molybdenum blue photometric method to determine the Si ion content, and use the weighing method to determine the amount of silica gel precipitation, and then conduct a comparison experiment.

\section{Dissolution rate of proppant by pre-acid test}

The dissolution of proppant in acid solution was tested by weight loss method under the condition of reservoir temperature and reaction time of $2 \mathrm{~h}$.

\section{Corrosion performance of pre-acid system test}

Without adding corrosion inhibitors, the corrosion rate of hydrochloric acid, mud acid, and polyhydroacid to N80 steel sheet and its own corrosion inhibition were measured, so no corrosion inhibitor was added in the experiment. The design time is $4 \mathrm{~h}$, the reaction is suspended, and the constant temperature of the water bath is $90{ }^{\circ} \mathrm{C}$. 
Mineral energy spectrum analysis after pre-acid reacts with rock

After grinding the rock, add acid water bath at $90{ }^{\circ} \mathrm{C}$ and react for $4 \mathrm{~h}$, take samples and wash them with distilled water repeatedly. Then, the composition and content of mineral elements before and after acidizing are determined by energy spectrum analysis, so as to judge the damage degree of acid to reservoir.

\section{Evaluation experiment of fracturing effect improved by pre-acid}

\section{Test of rock strength reduced by pre-acid damage}

Fracture pressure is very important for hydraulic fracturing, and pre-acid can effectively reduce formation fracture pressure (Peng et al. 2013). The mechanism of pre-acid in reducing formation fracture pressure has two aspects. First, the acid can not only clean mud pollution during drilling but also change the mineral content of the reservoir and the cementation strength of the pores, and reduce the rock cohesive force and friction angle; second, when the perforation holes are not clean, purifying the perforation holes can greatly reduce the rupture pressure. The pre-acid pretreatment technology reduces the fracture pressure. It not only changes the mechanical properties of the rock but also improves the liquid absorption capacity of the formation, thereby increasing the construction displacement and achieving the purpose of increasing the width of the joint.

Prikryl (2001) statistically studied the relationship between rock mineral content and composition, compressive strength and mechanical anisotropy. The statistical results show that the mineral composition is not the only factor that affects the mechanical properties of rocks. The arrangement of minerals and pore structure are also important factors that determine the mechanical properties of rocks. Dunning and Miller (1984) believes that the acid rock reaction changes the mechanical properties of rocks by changing the composition and structure of the rock and soil; Krajcinovic and Fonseka (1981) believes that the acid solution dissolves the cement between the calcareous particles, causing the stress concentration between the calcareous particles, thereby changing the carbon and the mechanical properties of acid rock; Qi et al (2020) studied the acid corrosion morphology of cracks and discussed the influencing factors and improvement methods of crack conductivity; Jiawei et al. (2020) conducted an acid fracturing experiment, using hydrochloric acid, guar gum, guar gum and acid as the fracturing fluid to analyze the characteristics of acid-etched cracks. The experimental results show that the acid has corroded the rock on the surface of the fracture, especially near the borehole. Acidic fluids easily open micro-cracks and pores and leak into these spaces, thereby shortening the propagation distance of initial acid corrosion cracks.
Acid reacts with rocks, especially with soluble minerals, which change the rock mineral parameters and greatly reduces the fracture pressure of the reservoir rock (Jin et al. 2020). We test the influence of different acid injection amount and concentration on rock microstructure and mechanical parameters by displacement experiment. Then, the microstructure was analyzed by SEM. The core flow test can macroscopically evaluate the effect of pre-acid on the improvement of formation core permeability. To investigate more on the microscopic changes in the rock structure after acid injection, the capillary flow porometry was used to measure and compare the pore throat size before and after the acid injection. By micro-displacement experiments of reservoir cores before and after acidification, the effective pore throat distribution before and after acidification is quantitatively described, and the change of rock pore throat structure before and after acidification is compared to evaluate the effect of acidification from a microperspective.

\section{Influence of pre-acid on gel breaking of fracturing fluid}

The molecular weight of ordinary guar gum is generally above 2 million and contains more than 4000 galactomannose-repeating units. The borate ions and the cis hydroxyl group in the guar gum molecule can form a relatively stable hydrogen bond through intermolecular cross-linking to form a high-viscosity jelly with excellent rheology. The derivatives of guar gum, such as hydroxypropyl guar gum, can be cross-linked with boron as long as the polymer chain contains a considerable proportion of cis hydroxyl groups. The cross-linking effect in the fracturing fluid is the borate ions, and the content of borate determines the degree of crosslinking of the fracturing fluid. According to the principle of chemical equilibrium movement, as long as the $\mathrm{pH}$ value of the fracturing fluid is lowered, the reaction direction can be moved to the left, and the degree of cross-linking can be controlled. After the completion of the pre-acid fracturing construction, the flowback of the acid liquid causes the $\mathrm{pH}$ value of the fracturing fluid to decrease, thereby reducing the concentration of borate in the fracturing fluid, and realizing the non-degradable gel breaking of the fracturing fluid.

Use the atomic force microscope to observe the microstructure of the fracturing fluid, determine the performance of the fracturing fluid and the changes caused by the acid to the microstructure of the fracturing fluid, and analyze the impact of the acid on the performance of the fracturing fluid.

\section{Influence of pre-acid on clay expansion and particle migration}

Due to the complexity of its chemical composition and characteristics, formation clay can cause clay swelling when it encounters foreign fluids such as water-based fracturing fluid 
filtrate. The high content of filamentous illite in the formation has potential for quick-sensitive damage. The clay stabilizer in the pre-acid solution can prevent clay from swelling. Using an acid solution system containing HF or other HF generated by the reaction as the pre-acid can react with clay minerals, reducing the number of swelling minerals and reducing the number of fast-sensitive minerals, thereby further reducing the damage of clay swelling and particle migration.

(1) Analysis of the composition of clay minerals before and after peracid

By using electron microscope scanning together with $\mathrm{X}$-ray diffraction to quantitatively analyze the composition of various minerals in the rock core, static observation of the internal structure of the core, an intuitive understanding on the situation after the acid reacts with the minerals can be developed

(2) Research on the influence of $\mathrm{pH}$ value on clay-swelling rate

The low pH value environment caused by the acid system is also beneficial in inhibiting the expansion of clay. Laboratory experiments were conducted to study the swelling rate of bentonite at different $\mathrm{pH}$ values to evaluate the effect of $\mathrm{pH}$ on the swelling rate.

The experiment took $5 \mathrm{ml}$ of bentonite placed in acid solutions of different $\mathrm{pH}$ values. The experiment temperature and time was $70{ }^{\circ} \mathrm{C}$ and $48 \mathrm{~h}$, respectively.

\section{Pre-acid cleaning matrix pore and support fracture test}

The acid solution can largely dissolve the fracturing fluid residue and polymerized filter cake, and further strengthen the gel breaking of the fracturing fluid, so as to achieve the function of cleaning and supporting cracks.

(1) Dissolution experiment of pre-acid on fracturing fluid filter cake.

During the fracturing process, the fracturing fluid in the fractures is continuously filtered out as the fracturing fluid is injected into the fractures. When the fractures are closed, the fracturing fluid in the fractures is further concentrated, resulting in a high concentration of HPG glue in the filter cake, and the breaker content is relatively low, the gel breaker does not work fully, so that the filter cake cannot be broken or the gel is not completely broken, which causes damage to the matrix permeability.

A $0.4 \%$ guar gum base fluid is prepared with conventional fracturing guar gum powder. After it is fully swelled, cross-linked guar gum is made, and a high temperature and high-pressure static fluid filter is used to simulate the formation of a filter cake during propped fracturing. The weight loss method was used to dissolve the filter cake with pre-acid at $75^{\circ} \mathrm{C}$ for $2 \mathrm{~h}$.

(2) Pre-acid cleaning experiment for matrix pores

The cross-linked fracturing fluid has a high viscosity. Consequently, it cannot pass through the core under the experimental conditions. Therefore, an uncross-linked fracturing fluid is selected for the experiment. During the experiment, the acid solution was reacted with the reservoir rock powder for $2 \mathrm{~h}$ at the reservoir temperature and filtered to obtain the residual acid solution.

(3) Cleaning experiment of supporting cracks by acid

First, use the base fluid to measure the permeability of the artificial core; then inject the cross-linked fracturing fluid; after the fracturing fluid breaks the gel, use the base fluid to measure the permeability of the artificial core; inject the residual acid (the preparation method is the same as before), use the base fluid to measures the permeability of the artificial core and evaluate the degree of improvement on the permeability of the residual acid.

(4) Microscopic analysis of core damage

Infrared spectroscopy is one of the most commonly used methods to identify compounds and determine the molecular structure. It can perform qualitative or quantitative analysis on a single component or each component in a mixture. Using infrared spectroscopy to produce characteristic adsorption peaks for the polymer in the fracturing fluid to evaluate the fracturing fluid's adsorption of rock dust and the removal of acid damage to the fracturing fluid.

\section{Results and discussion}

\section{Test results and analysis of hydraulic fracturing damage}

\section{Evaluation results and analysis of fracturing fluid dynamic adsorption retention damage}

Taking the concentration of hydroxypropyl guar gum in the solution as the abscissa and the permeability damage rate as the ordinate, the experimental data are shown in Fig. 3. After the experiment, the hydroxypropyl guar gum solution was subjected to ultraviolet spectroscopy to obtain the residual hydroxypropyl guar gum content in the solution, and then the retention of the hydroxypropyl guar gum in the proppant placement belt was obtained.

The experimental results in the figure show that as the concentration of hydroxypropyl guar gum increases, the retention of hydroxypropyl guar gum in the ceramsite proppant belt gradually increases, and also the damage to the permeability of the proppant belt gradually increases. When

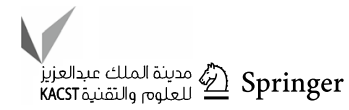




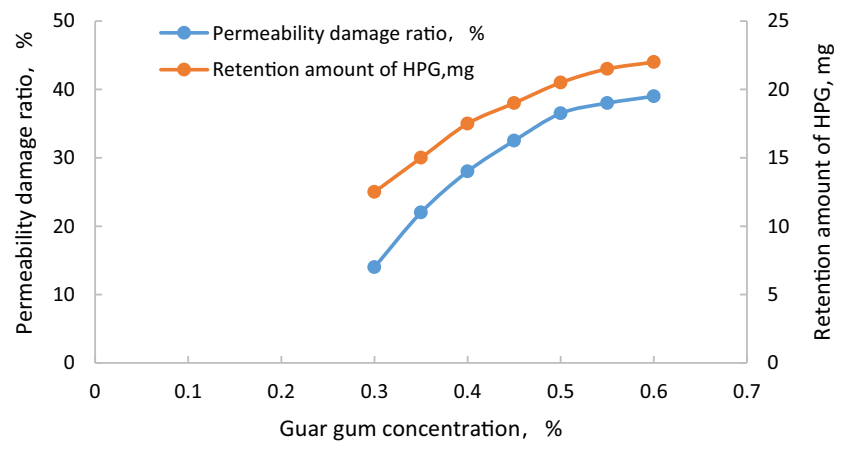

Fig. 3 The dynamic adsorption retention and permeability loss rate curve of hydroxypropyl guar gum with the concentration of guar gum

increased, the penetration rate drops by $40 \%$. The retention volume increased rapidly in the early stage from $13.7 \mathrm{mg}$ to $20.5 \mathrm{mg}$. After the hydroxypropyl guar gum concentration reached $5000 \mathrm{mg} / \mathrm{L}$, the retention volume slowly increased to saturation. The increasing trend of penetration rate of damage is roughly consistent with the amount of retention. First, it rapidly increased from 14 to $36 \%$, and then slowly increased to $39 \%$ to maintain stability.

\section{Observation and analysis of electron microscope}

From the scanning electron microscope image, it can be seen that in the ceramsite proppant placement belt after the use of hydroxypropyl guar gum solution, there is indeed the phenomenon of hydroxypropyl guar gum molecular chain retention and molecular flocculation blocking the pore throat.

From the overall comparison in Fig. 4a, there are a large number of hydroxypropyl guar gum molecules on the surface and intergranular pores of the ceramsite after injury, which has two forms of chain and network structures, occupying a large number of pores; namely, flow channels. By further enlargement, as shown in Fig. 4b, the intergranular pores before damage have a larger pore size and better flow ability. After the hydroxypropyl guar gum solution is displaced, the guar gum molecular chains are entangled, curled and form flocs to stay in the pores, which seriously affect the conductivity of the cracks. In addition, the degree of molecular chain entanglement is complicated, and the flocs are stable in shape and large in size, and it is difficult to be discharged back. Enlarged to a ceramsite as shown in Fig. 4c, the surface of the ceramsite is uneven before injury; however, there is material retention. After the injury, the hydroxypropyl guar gum molecule with a network structure is obviously covered on the surface of the ceramsite. There are also some small-area flake guar gums. Although these hydroxypropyl guar gum molecules covering the surface do not significantly block the pore throats, they are present in almost all ceramsite particles, which have a positive effect on the reduction in the pore volume of the entire porous medium and also cause proppant spreading. One of the main factors contributing to the decline of belt penetration. Enlarging the surface of a single ceramsite as shown in Fig. 4d, it can be seen that not only flakes and net-like hydroxypropyl guar gum stay on the surface of the ceramsite, but also chain-like entanglement. Due to the uneven surface of the ceramsite, the chain guar gum polymer is easy to hang on the surface. However, the contact force between them is very weak, and it is easy to be washed out of the proppant placement zone under a longer flowback time or a strong flowback displacement.

\section{Test results and analysis of damage of fracturing fluid filtrate to core matrix and microfracture}

The experimental results are shown in Fig. 5. The damage of filtrate to the matrix is not very strong, the core permeability damage rate is $11.85-39.81 \%$, and the average damage degree is $24.64 \%$.

Using the same experimental process described above, the fracturing fluid damage experiment was conducted on the core filled with proppant. The experimental results are shown in Fig. 6. The damage of fracturing fluid to supporting fractures is higher than that to the core matrix, and the average damage rate of fractures is $47.6 \%$.

It can be seen from the experimental results that the damage of fracturing fluid to the sand-filled cracks is more than $40 \%$, and the maximum is $60 \%$, indicating that the fracturing fluid seriously damages the sand-filled cracks.

\section{Design results and analysis of pre-acid system}

\section{Corrosion rate test results}

The test results are shown in Fig. 7.

According to the evolution of rock dust dissolution rate with an acid concentration, and at the same time, it is considered that the pre-acid stays in the formation for a long time during the pre-acid fracturing construction process. Therefore, it is necessary to ensure that the acid solution effectively dissolves the rock and minerals and compares it, and also maintains a low $\mathrm{pH}$ value for a long time without causing secondary damage to the formation due to excessive acid concentration.

\section{Evaluation results and analysis of retarded acid energy}

(1) $\mathrm{HCl}$ and SA601 acidity curve analysis.

From Fig. 8, the acidity curve of $\mathrm{HCl}$ has only one mutation point, and the mutation part of the curve is very steep, almost a straight line, indicating that $\mathrm{HCl}$ is a strong monobasic acid, and $\mathrm{H}^{+}$is in a fully ionized state in the solution. The acidity curve of SA601 


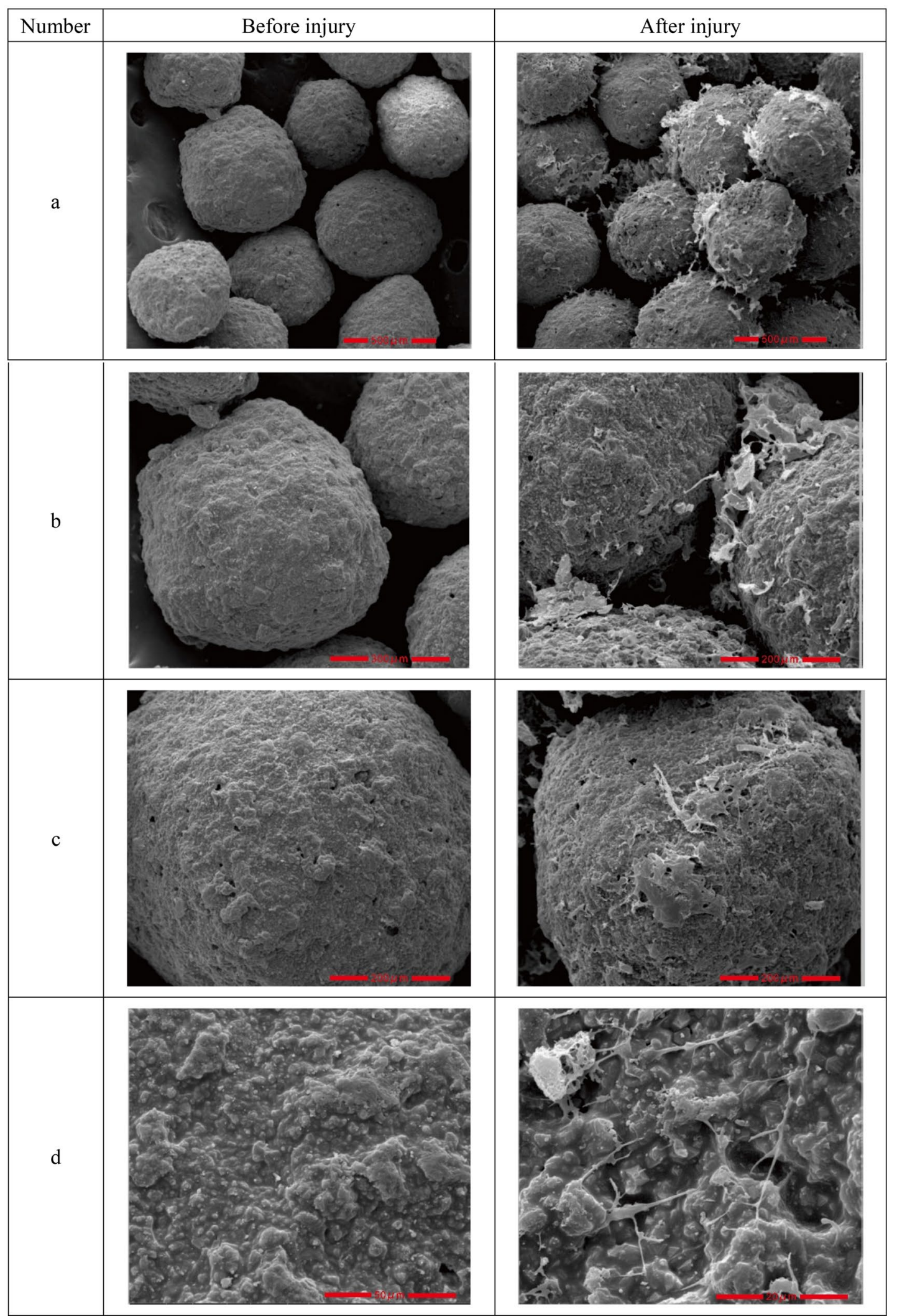

Fig. 4 The microscopic morphology of fracturing fluid adsorbed and retained on the surface of the proppant 


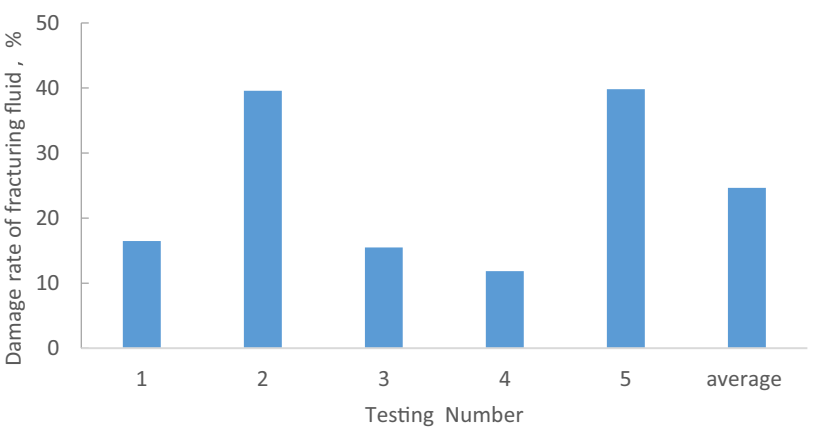

Fig. 5 Damage of fracturing fluid to the core matrix

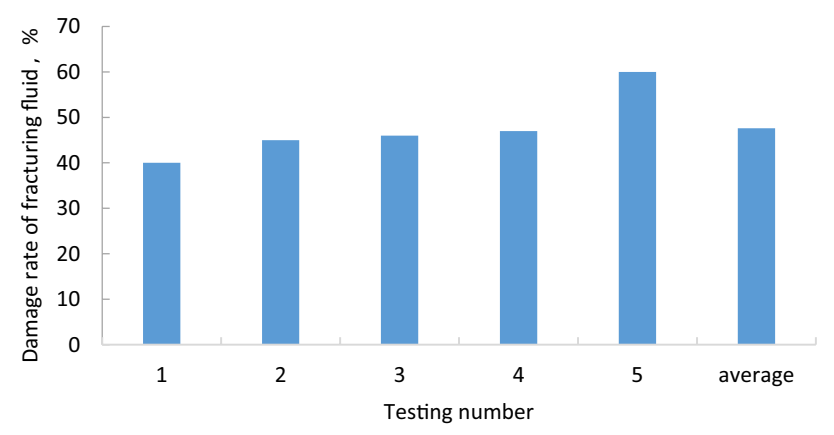

Fig. 6 Damage of fracturing fluid to supporting fractures

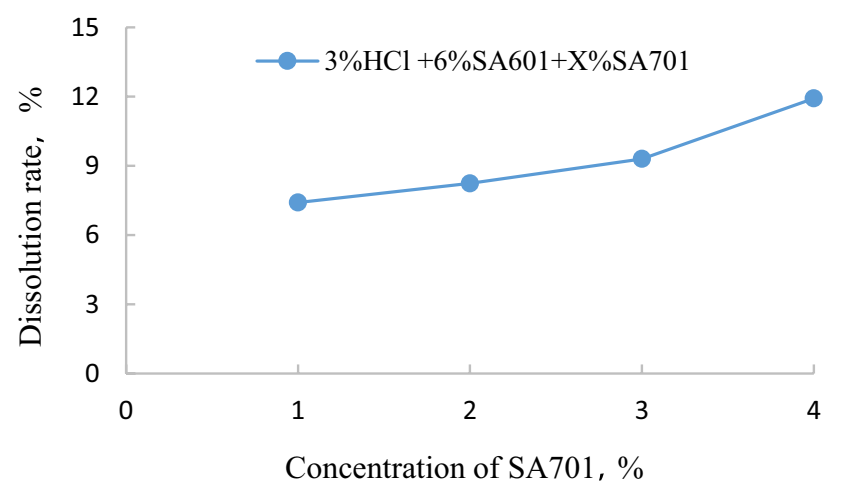

Fig. 7 Change of rock dust dissolution rate with acid concentration

has multiple mutation points, and the mutation part is smooth, indicating that SA601 is a polybasic weak acid, $\mathrm{H}^{+}$is partially ionized in the solution, and there will be $\mathrm{H}^{+}$in the solution as $\mathrm{H}^{+}$is consumed during the process of adding $\mathrm{NaOH}$, it gradually ionizes to achieve ionization equilibrium. Comparing the acidity curves of $\mathrm{HCl}$ and $\mathrm{SA} 601$ shows that the initial $\mathrm{pH}$ value of $\mathrm{HCl}$ is lower than that of SA601, that is to say $\left[\mathrm{H}^{+}\right]$in $\mathrm{HCl}$ solution is higher than SA601. SA601 will gradually re-ionize $\mathrm{H}^{+}$with the consumption of $\mathrm{H}^{+}$, while $\mathrm{HCl}$ will no longer ionize $\mathrm{H}^{+}$.

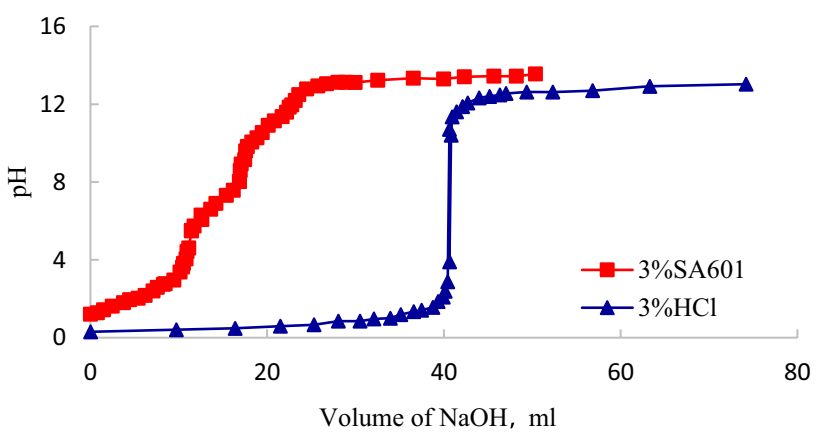

Fig. 8 Comparison of acidity curve between 3\% SA601 and 3\% $\mathrm{HCl}$

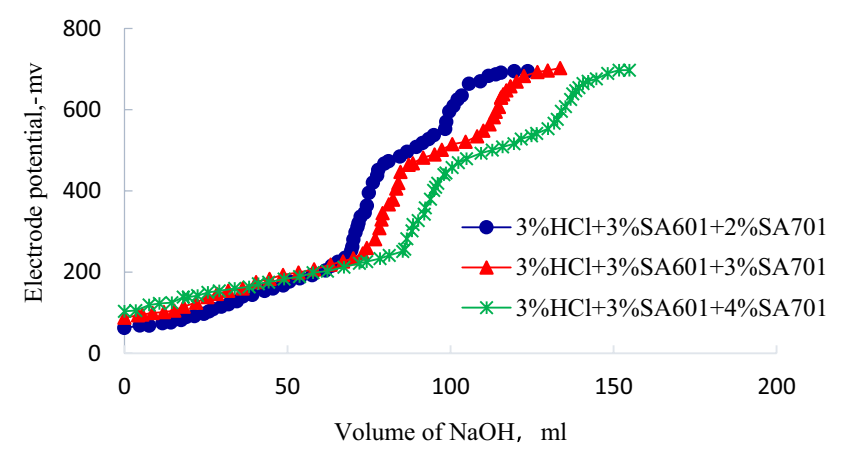

Fig. 9 Potential curves of different concentrations of polyhydrogen acid

(2) Analysis of acidity curve of polyhydrogen acid system.

The electrode potential measured by the antimony electrode indirectly expresses $\left[\mathrm{H}^{+}\right]$, that is, indirectly expresses the $\mathrm{pH}$ value of the solution. From Fig. 9, $\mathrm{H}^{+}$ of the polyhydrogen acid system is also ionized step by step, and the curve change is relatively smooth, indicating that the polyhydrogen acid also has buffering properties. Also, the figure shows that the initial $\mathrm{pH}$ of the solution increases with the increase of SA701, indicating that SA701 has also buffering properties.

\section{Test results and analysis of inhibiting secondary precipitation}

(1) Suppresses metal ion precipitation.

Figure 10 shows that solution $b$ is a clear and transparent solution. Indicating that after the polyhydrogen acid chelates $\mathrm{Ca} 2+$, no precipitation is formed under acidic conditions, and the polyhydrogen acid liquid system has good scale inhibition performance; after the solution is neutral, the solution is no longer transparent but does not delaminate, showing that the polyhydrogen acid still has a certain dispersion ability. 


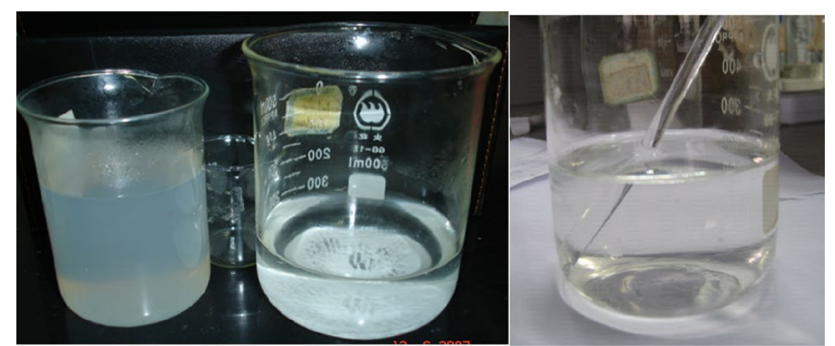

Fig. $10 \mathbf{a}, \mathbf{b}$, and $\mathbf{c}$ solution

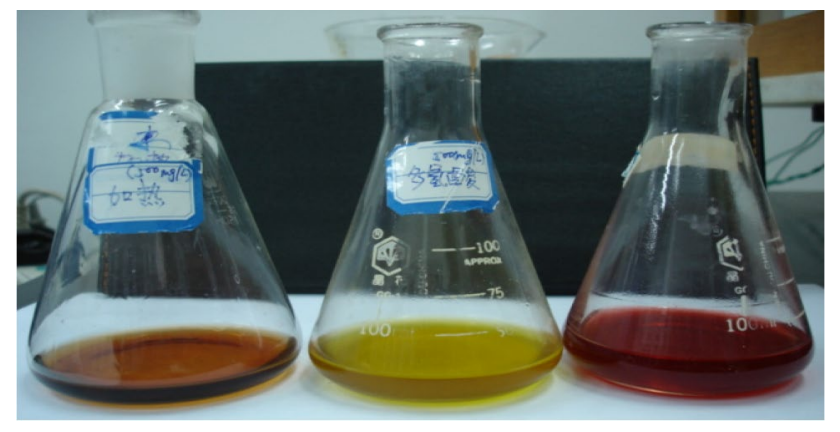

Take a certain amount of a, $c$, $d$ solution and add calcium red indicator to observe the color of the solution. From the three solution colors in Fig. 11, it can be seen that there is $\mathrm{Ca}^{2+}$ in both solutions $\mathrm{c}$ and $\mathrm{d}$, and there is none in the solution a, indicating that polyhydrogen acid affects metal ions. It has good chelating ability, which can effectively inhibit the precipitation of fluorosilicate.

(2) Inhibit silica gel precipitation.

The experimental results are shown in Table 1.

Table 1 shows that the chelating agent can effectively inhibit the formation of silica gel precipitation.

Additionally, as shown in the comparison chart before and after adding a chelating agent to the Si ion standard solution (Fig. 12), a large amount of precipitation is generated after heating the sample without chelating agent for $30 \mathrm{~min}$, and the amount of precipitation increases with the heating time. Although the sample without chelating agent has high Si ion concentration, the liquid is still very transparent after heating for $90 \mathrm{~min}$, and the transparency of the liquid after heating for $120 \mathrm{~min}$ decreases and starts to precipitate.

Fig. $11 \mathbf{c}, \mathbf{a}$ and $\mathbf{b}$ solution of involve calred tracer agent

Table 1 Chelating agents inhibit silica gel precipitation

\begin{tabular}{|c|c|c|c|c|c|c|}
\hline \multirow[t]{2}{*}{ Sample } & \multicolumn{2}{|l|}{$30 \mathrm{~min}$} & \multicolumn{2}{|l|}{$90 \mathrm{~min}$} & \multicolumn{2}{|l|}{$120 \mathrm{~min}$} \\
\hline & $\begin{array}{l}\text { Si con- } \\
\text { centration } \\
(\mathrm{mg} / \mathrm{l})\end{array}$ & $\begin{array}{l}\text { Silica gel } \\
\text { precipitation } \\
(\mathrm{mg})\end{array}$ & $\begin{array}{l}\text { Si con- } \\
\text { centration } \\
(\mathrm{mg} / \mathrm{l})\end{array}$ & $\begin{array}{l}\text { Silica gel } \\
\text { precipitation } \\
(\mathrm{mg})\end{array}$ & $\begin{array}{l}\text { Si con- } \\
\text { centration } \\
(\mathrm{mg} / \mathrm{l})\end{array}$ & $\begin{array}{l}\text { Silica gel } \\
\text { precipitation } \\
(\mathrm{mg})\end{array}$ \\
\hline $\begin{array}{l}\text { Without } \\
\text { chelat- } \\
\text { ing agent } \\
(2000 \mathrm{mg} / \mathrm{l})\end{array}$ & 1243.12 & 129.48 & 765.29 & 211.22 & 586.44 & 241.82 \\
\hline $\begin{array}{l}\text { Add chelat- } \\
\text { ing agent } \\
(4000 \mathrm{mg} / \mathrm{l})\end{array}$ & 3810.63 & 32.39 & 3613.13 & 66.18 & 3020.63 & 167.54 \\
\hline
\end{tabular}

Fig. 12 Comparison diagram of $\mathrm{Si}$ ion standard solution before and after adding chelating agent
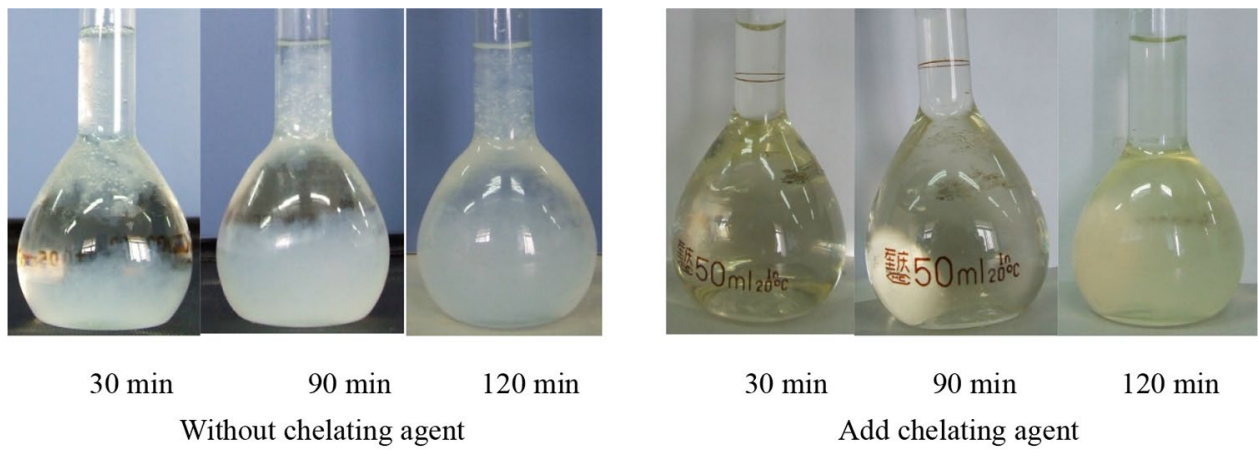


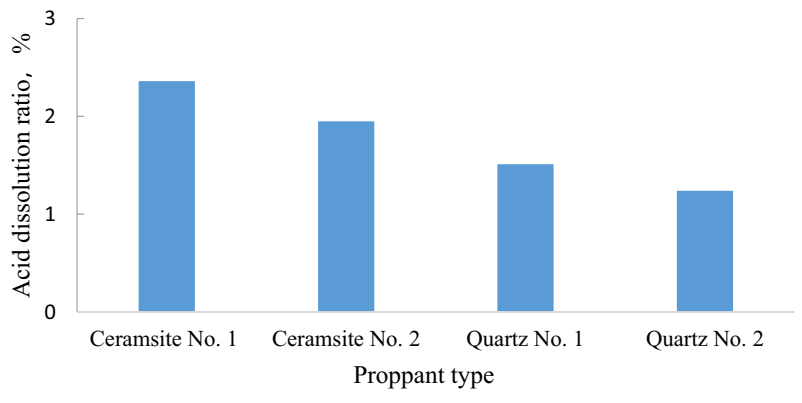

Fig. 13 Acid solution to proppant dissolution rate

\section{Dissolution rate of proppant by pre-acid}

As shown in Fig. 13, simulating the formation temperature conditions and conducting research on the dissolution rate of proppant by acid shows that the dissolution rate of the proppant by the acid solution is very low, and the acid treatment will not affect the proppant support capacity.

\section{Test results and analysis of corrosion performance of pre-acid system}

The test results are shown in Table 2.

Figure 14 shows a photograph of the corrosion of the steel sheet by the acid solution (the left is the photograph before corrosion, and the right is the photograph after corrosion).

It can be seen from the test results that at $90{ }^{\circ} \mathrm{C}$, under normal pressure and static conditions, $12 \% \mathrm{HCl}+3 \% \mathrm{HF}$ and $8 \%$ hydrochloric acid will corrode the $\mathrm{N} 80$ steel sheet more severely. Corrosion is very weak.

\section{Energy spectrum test results and analysis of minerals after reaction between pre-acid and rock}

See Table 3 for determination.

After the acid reacts with the rock, the mineral composition of the rock will inevitably change. The composition of the rock and mineral before and after acidification is analyzed through the energy spectrum to judge the change of
Table 2 Normal pressure static corrosion test results

\begin{tabular}{llcc}
\hline Number & Acid $(280 \mathrm{ml})$ & Temperature reflex $\left({ }^{\circ} \mathrm{C}\right)$ & $\begin{array}{l}\text { Corrosion } \\
\text { rate }\left(\mathrm{g} / \mathrm{m}^{2} \mathrm{~h}\right)\end{array}$ \\
\hline $\mathrm{a}$ & $10 \% \mathrm{HCl}$ & 90 & 432.89 \\
$\mathrm{~b}$ & $12 \% \mathrm{HCl}+3 \% \mathrm{HF}$ & 90 & 2097.70 \\
$\mathrm{c}$ & $3 \% \mathrm{HCl}+3 \% \mathrm{SA} 601+2 \% \mathrm{SA} 701$ & 90 & 8.15 \\
$\mathrm{~d}$ & $3 \% \mathrm{HCl}+3 \% \mathrm{SA} 601+3 \% \mathrm{SA} 701$ & 90 & 11.58 \\
\hline
\end{tabular}

Fig. 14 Corrosion photograph of steel sheet

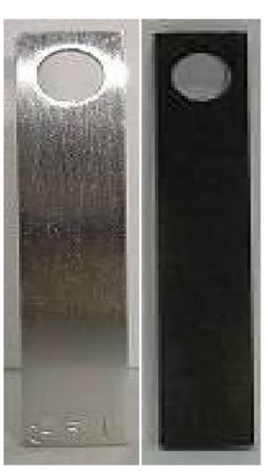

a

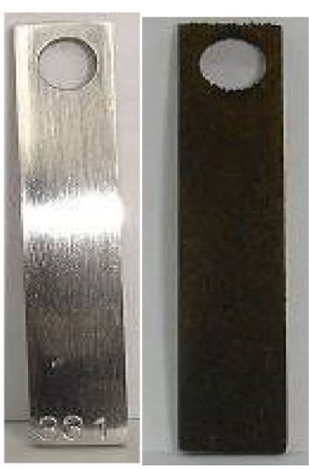

b

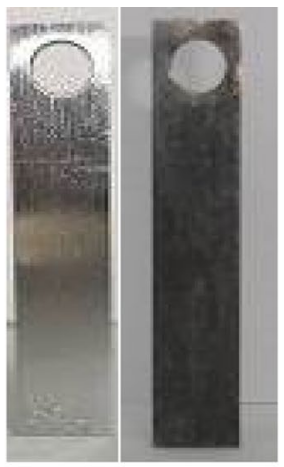

c

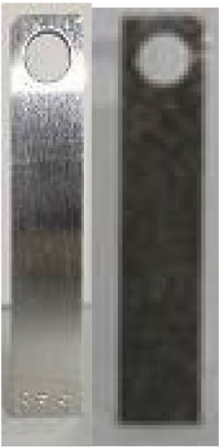

d
Table 3 Determination of solid phase element composition after the reaction of each pre-acid with feldspar

\begin{tabular}{lllllllll}
\hline & \multicolumn{1}{l}{ wt\% } & & & & & & & \\
\cline { 2 - 9 } Element & $\mathrm{C}$ & $\mathrm{O}$ & $\mathrm{Na}$ & $\mathrm{Al}$ & $\mathrm{Si}$ & $\mathrm{K}$ & $\mathrm{Ca}$ & $\mathrm{F}$ \\
\hline Primitive minerals & 4.54 & 40.83 & 6.68 & 11.03 & 33.28 & 2.45 & 1.19 & 0 \\
After mud acid reaction & 3.01 & 40.79 & 1.77 & 3.17 & 48.34 & 0 & 0 & 2.91 \\
After polyhydroacid reaction & 3.47 & 40.88 & 5.96 & 9.50 & 37.93 & 1.65 & 0 & 0.61 \\
\hline
\end{tabular}


the acid and mineral reaction elements, and to compare the damage degree of different acid on the rock. It can be seen from the above experimental results that there is no $\mathrm{F}$ element in the original minerals, but $\mathrm{F}$ elements are produced in the minerals after the acid reaction, which shows that all sandstones will cause more or less damage after acidification, but it is only harmful. The size is different. Compared with the acid solution system containing F element, polyhydrogen acid is obviously less harmful than the mud acid.

\section{Experimental results and analysis of pre-acid improving fracturing effect evaluation}

\section{Test results and analysis of pre-acid reducing rock strength}

(1) Measurement results and analysis of rock mechanical properties.

(a) The influence of acid concentration.

The influence of different acid concentrations and different acid concentrations of the same acid concentration on rock mechanical parameters (Young's modulus and breaking ratio) under the same acid injection rate (50PV) is shown in Figs. 15 and 16.

It can be seen from Figs. 15 and 16 that as the acid concentration and the amount of acid injection increase, the Young's modulus and Poisson's ratio of the rock will also increase. Acidification pretreatment is effective in reducing the fracture pressure of the reservoir rock. However, when choosing the amount and strength of the acid solution, the selection must be made carefully to prevent the rock skeleton from collapsing.

(b) Stress-strain curve.

Figure 17 is the stress-strain curve of the rock sample after acid treatment. It can be seen that the strength of the rock decreases after treatment. As the acid liquid dissolves part of the minerals in the rock, the porosity of the rock increases. In the initial stage of compression, there is an obvious compaction stage. In the fail-

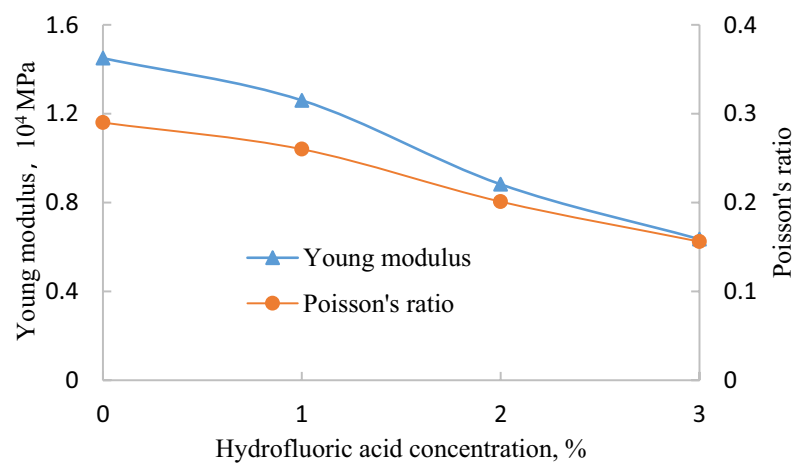

Fig. 15 Effect of HF concentration on Young modulus and Poisson's ratio

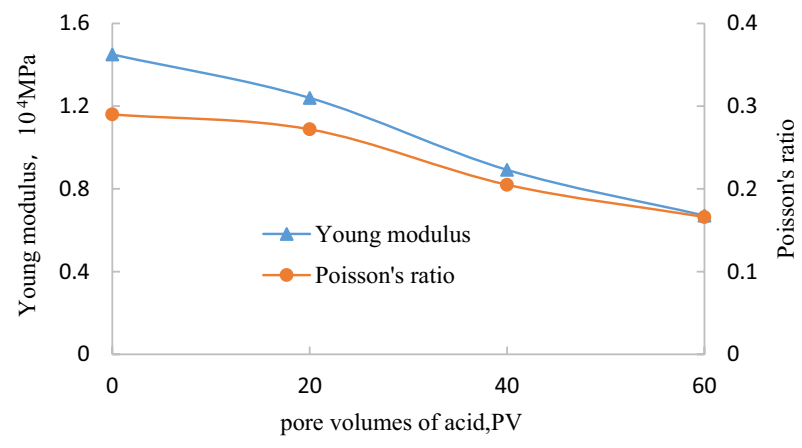

Fig. 16 Effect of acid volumes on Young modulus and Poisson's ratio

ure stage of the rock, the axial deformation is greater than the axial deformation of the rock without acid damage.

(2) SEM test results and analysis.

The SEM photograph before acid injection is shown in Fig. 18. It can be seen that the rock is relatively dense with occasional intergranular pores. Most of the primary intergranular pores are filled by chlorite, kaolinite or authigenic quartz, with a small number of intracrystalline dissolved pores.

The SEM picture after acid injection is shown in Fig. 19. It can be seen that by reacting with the rock, the acid can dissolve the soluble and soluble minerals in the rock, and produce some intergranular pores and crystal-dissolved pores, which to an extent increase the rock pores and change the connectivity of the rock pores.

(3) Evaluation results and analysis of micro-pore structure.

The pore throat distribution of different cores before and after acidification is shown in Fig. 20.

After acid treatment, the acid liquid dissolves the fillings in the pore throat and part of the rock skeleton. Consequently, the effective pore throat number and diameter of the core are increased, which indicates

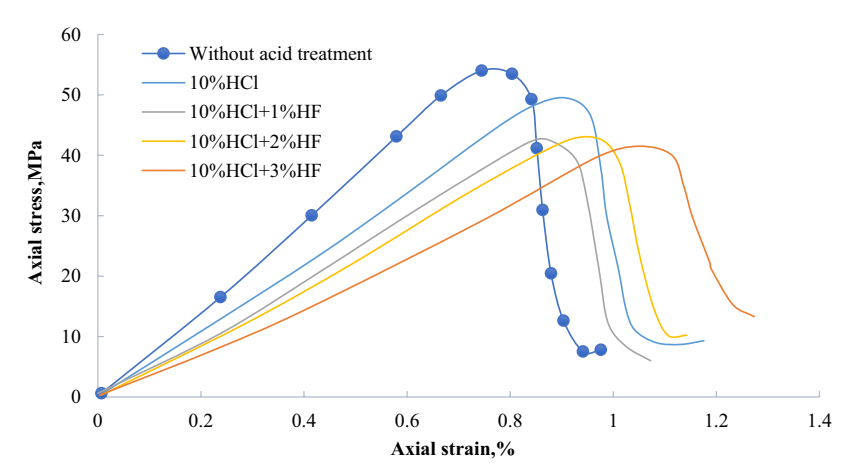

Fig. 17 Strain versus stress curve of rock after acid treatment

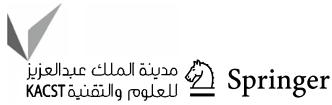


Fig. 18 SEM picture before acidification, $\mathbf{a}$ the rock is denser, with occasional intergranular pores, $\mathbf{b}$ the intergranular pores are filled with a large number of clay minerals, $\mathbf{c}$ clay minerals are wrapped around the substrate

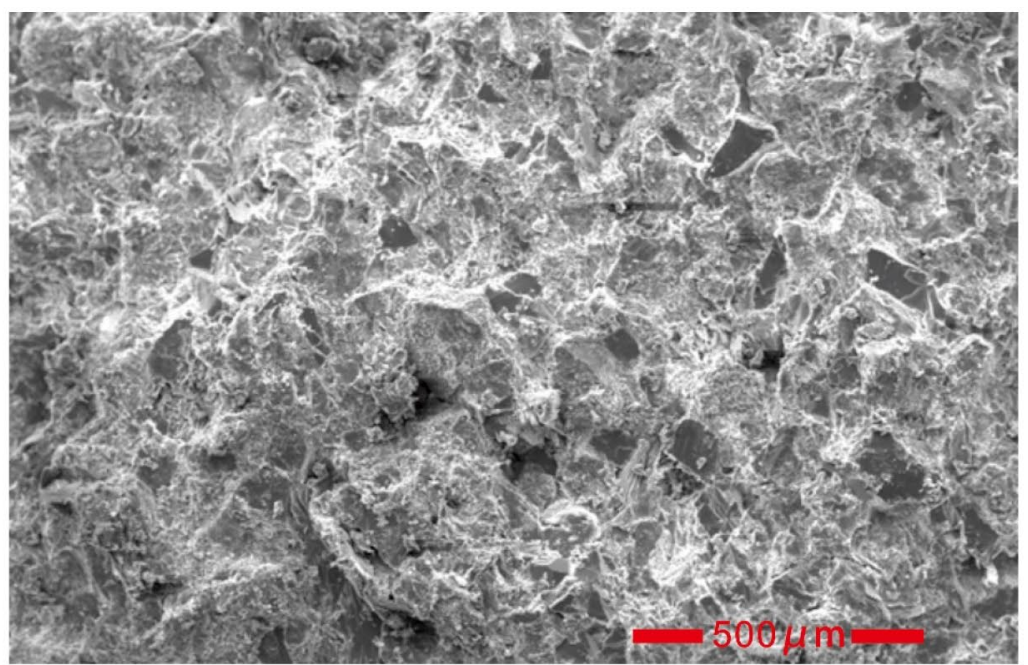

a The rock is denser, with occasional intergranular pores;

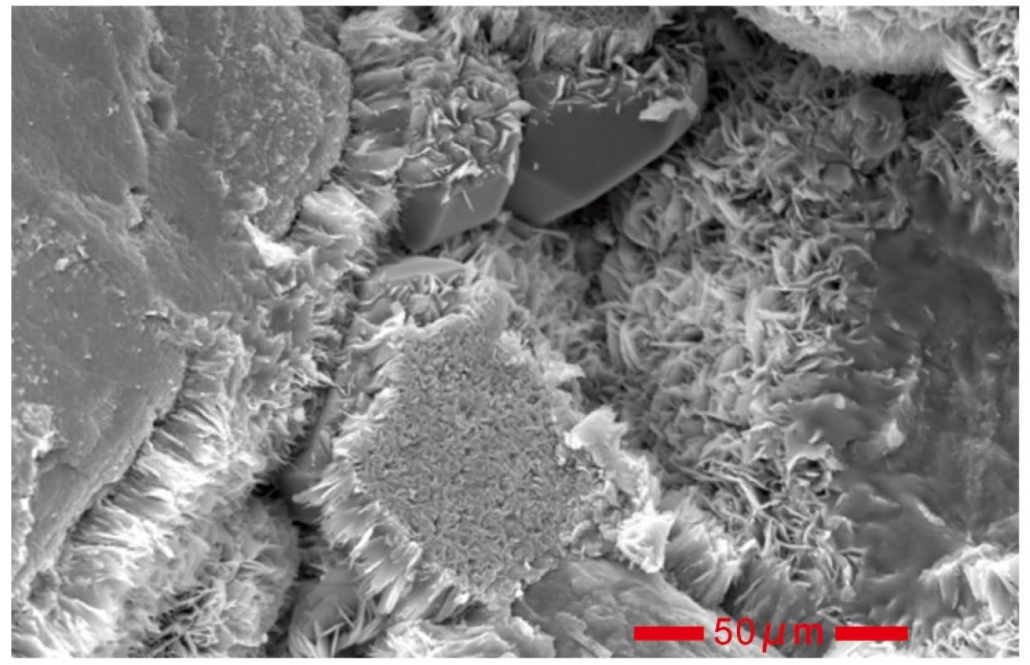

b The intergranular pores are filled with a large number of clay minerals;

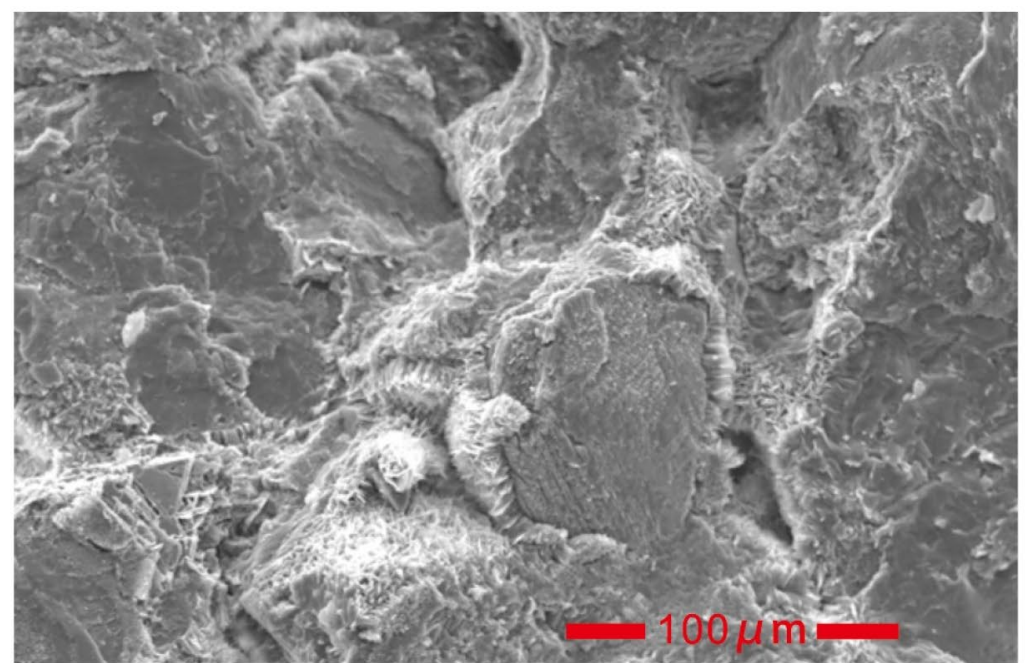

c Clay minerals are wrapped around the substrate. 
Fig. 19 SEM image after acidification, a acid dissolution holes are clearly visible, $\mathbf{b}$ partially insoluble clay minerals, $\mathbf{c}$ feldspar has crystal pores

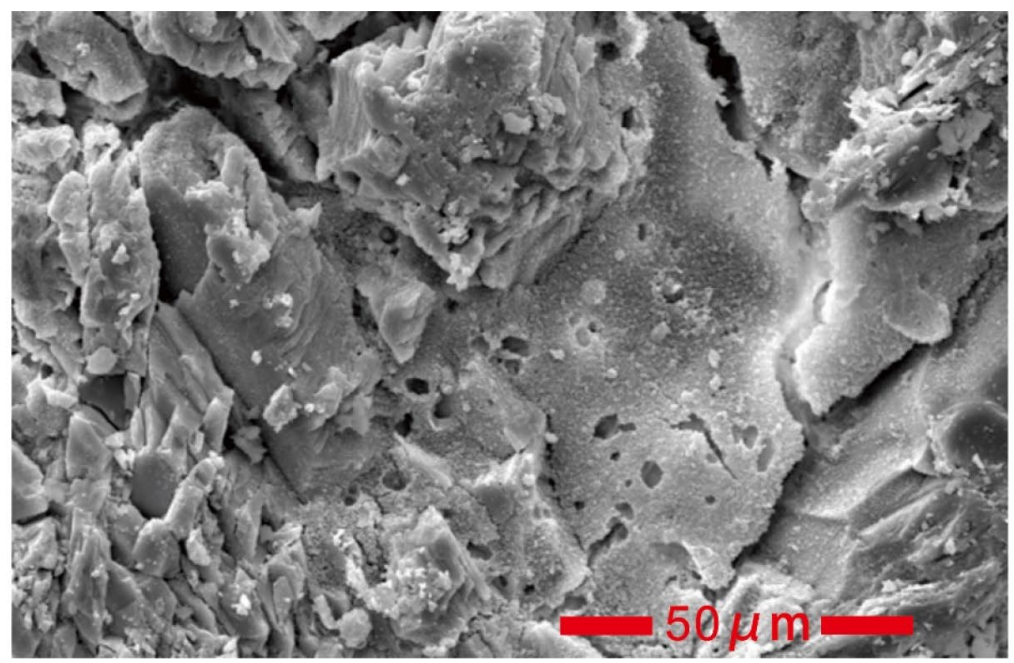

a Acid dissolution holes are clearly visible;

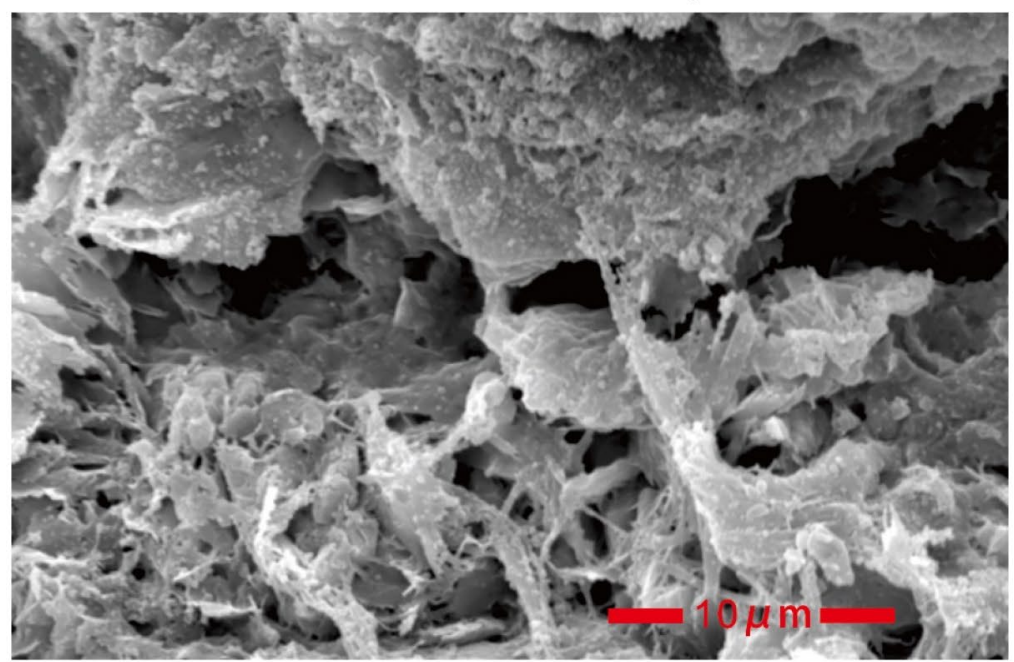

b Partially insoluble clay minerals;

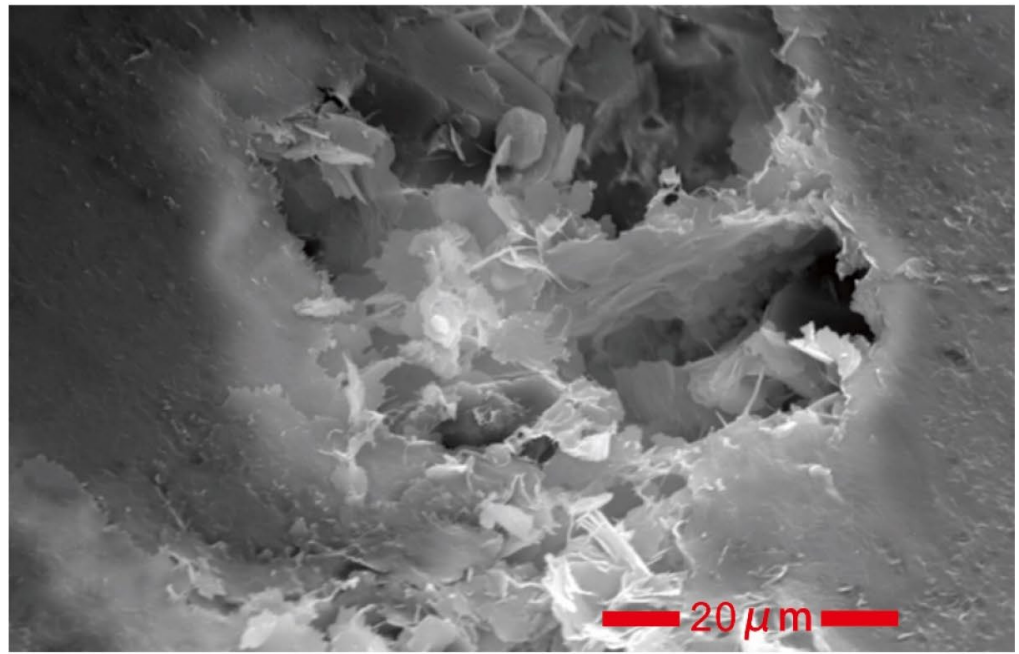

c Feldspar has crystal pores. 


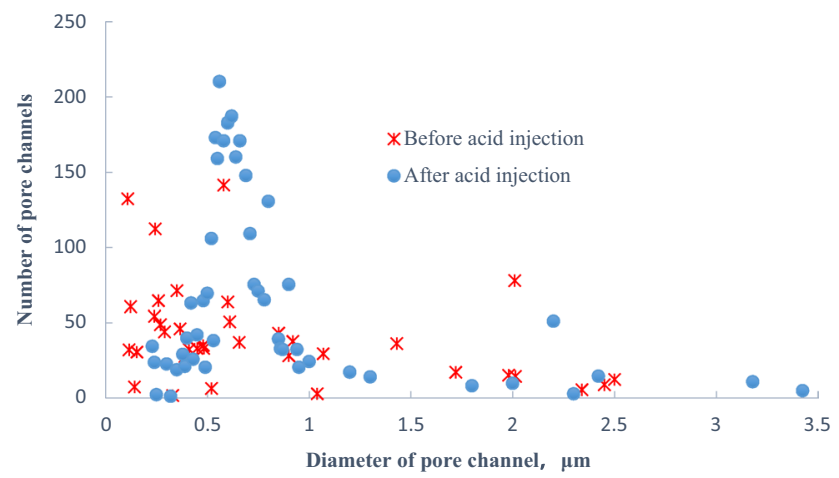

Fig. 20 Comparison of the distribution of the front pore throat before and after core acid treatment that the acidification played a role in improving the permeability of the core.

\section{Experimental results and analysis of the effect of pre-acid on viscosity expansion}

See Table 4.

\section{Experimental results and analysis of pre-acid reducing clay expansion and particle migration}

(1) Analysis of the composition of clay minerals before and after peracid

Table 4 Microstructure analysis results of fracturing fluid before and after encountering acid

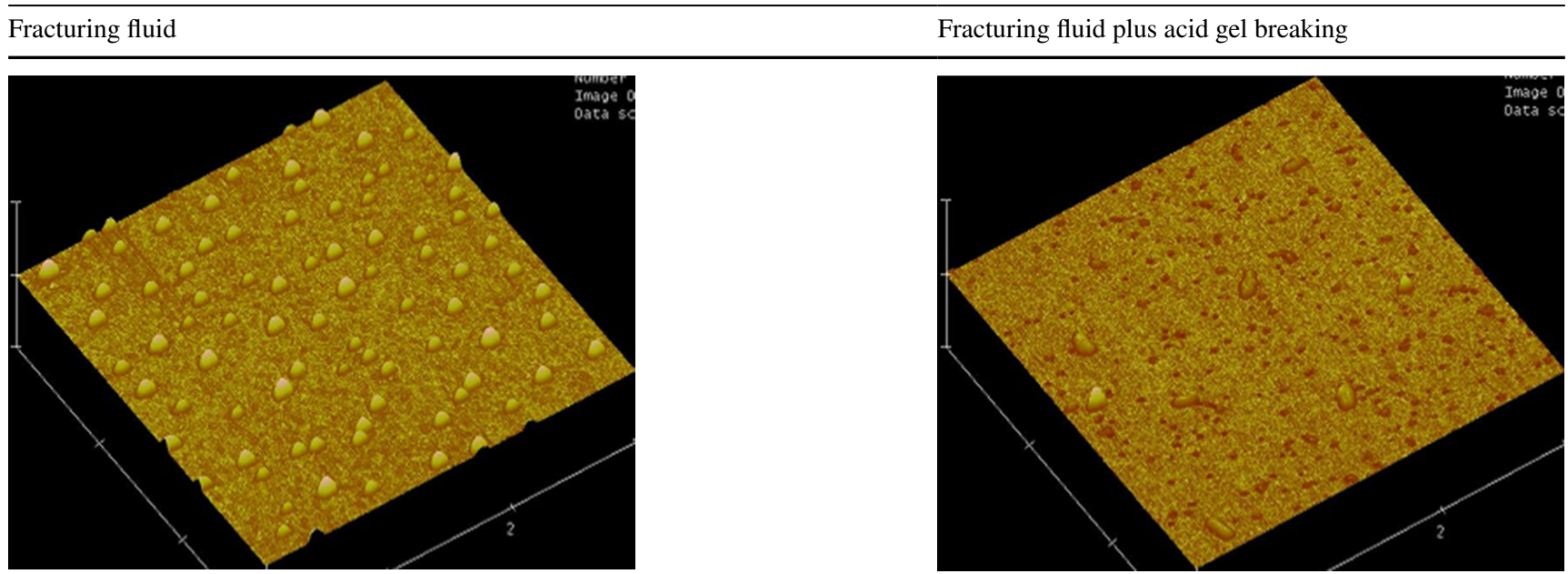

It is obvious from the three-dimensional diagram of the fracturing fluid that the molecules of the fracturing fluid after adding acid are obviously smaller
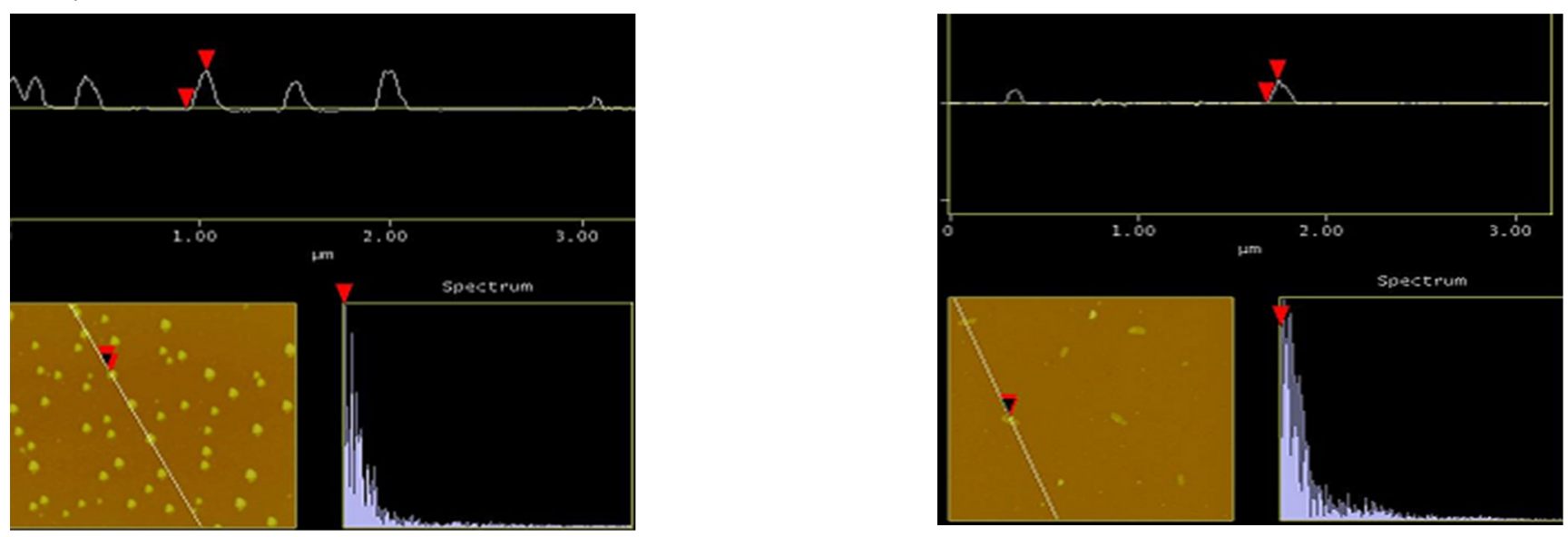

It can be seen from the molecular size data that the number and size of fracturing fluid molecules after acid have become smaller 
Table 5 XRD analysis results of core before and after peracid (whole rock analysis)

\begin{tabular}{|c|c|c|c|c|c|}
\hline & \multicolumn{5}{|c|}{ Nonclay minerals $(>4 \mu)$} \\
\hline & \multirow[t]{2}{*}{ Absolute content } & \multicolumn{4}{|c|}{ Relative content } \\
\hline & & Quartz & Potash feldspar & Plagioclase & Calcite \\
\hline Before reaction & 91.61 & 26.52 & 17.53 & 54.4 & 1.53 \\
\hline \multirow[t]{4}{*}{ After reaction } & 95.33 & 30.47 & 18.06 & 51.47 & 0.00 \\
\hline & \multicolumn{5}{|c|}{ Nonclay minerals $(<4 \mu)$} \\
\hline & \multirow[t]{2}{*}{ Absolute content } & \multicolumn{4}{|c|}{ Relative content } \\
\hline & & Kaolinite & Chlorite & Illite & $\begin{array}{l}\text { Iraqi/ } \\
\text { Mongolian } \\
\text { layer }\end{array}$ \\
\hline Before reaction & 8.39 & 77.69 & 11.28 & 5.01 & 6.03 \\
\hline After reaction & 4.67 & 83.20 & 9.15 & 2.15 & 5.51 \\
\hline
\end{tabular}

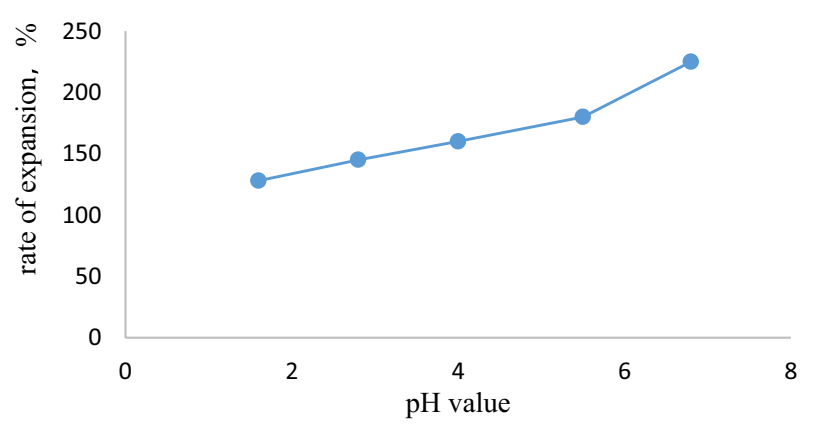

Fig. 21 Swelling rate of bentonite at different $\mathrm{pH}$ values

Table 5 shows the mineral composition analysis results of the rock samples before and after acid treatment.

Clearly, the results indicate that the absolute content of clay minerals is greatly reduced after the reaction. All the calcite is dissolved; however, only a small part of the chlorite, illite, and interlayer between illite and Mongolian remains. After the reaction, the clay minerals are mainly kaolinite, and most of the minerals that make the reservoir sensitive are consumed by the reaction.

(2) Research on the influence of $\mathrm{pH}$ value on clay-swelling rate

The experimental results are shown in Fig. 21.

The above figure indicates that the stronger the acidic environment, then the better it inhibits clay expansion. At the same time, clay and iron ion stabilizers are added to the acid solution to prevent the secondary precipitation of iron ions and the expansion and migration of clay minerals. Hence, improving the flowback rate of residual liquid and reducing the damage of residual liquid in the reservoir.
Table 6 Experimental results of dissolution of filter cake by acid solution $\left(75^{\circ} \mathrm{C}\right)$

\begin{tabular}{llll}
\hline Acid & $\begin{array}{l}\text { Weight before } \\
\text { filter cake test } \\
(\mathrm{g})\end{array}$ & $\begin{array}{l}\text { Weight after } \\
\text { filter cake test } \\
(\mathrm{g})\end{array}$ & Corrosion rate\% \\
\hline Polyhydroacid & 2.8762 & 2.5722 & 10.57 \\
\hline
\end{tabular}

The acid solution is obtained by reacting the rock dust and the acid solution at the reservoir temperature for $2 \mathrm{~h}$ after filtering

\section{Results and analysis of pre-acid cleaning matrix pores and supporting fractures}

(1) Experimental results and analysis of pre-acid dissolving fracturing fluid filter cake.

The experimental results are shown in Table 6.

It can be seen that the residual acid after $2 \mathrm{~h}$ of reaction still has good dissolution performance on the fracturing fluid filter cake, which can better relieve the damage of the fracturing fluid filter cake to the permeability.

(2) Experimental results and analysis of pre-acid cleaning matrix pores.

The relationship curve between $\mathrm{K} / \mathrm{K} 0$ of core damage and plugging removal effect test and pore volume multiple PV is shown in Fig. 22.

The experiment is divided into two parts. The first part considers the core damage, while the second part considers the plugging effect of acid on the core. The core damage experiment indicates that the fracturing fluid entering the reservoir will inevitably cause damage to the reservoir. The test is judged by the core permeability change, combined with the previous infrared spectrum experiment analysis, it shows that the fractur- 
Fig. 22 Experimental diagram of core damage and plugging removal

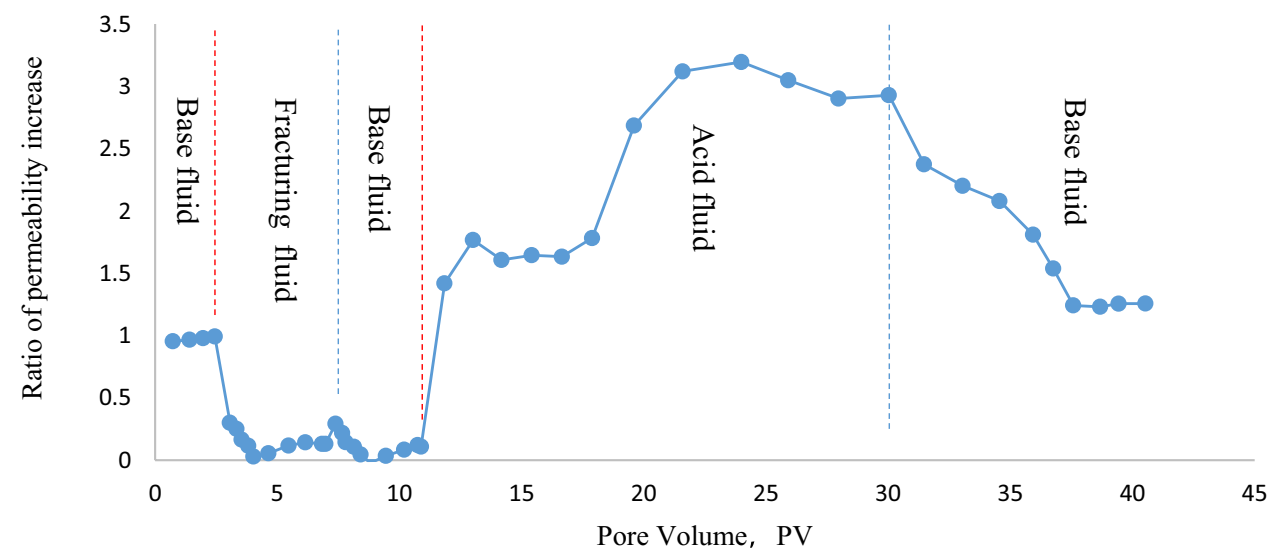

ing fluid entering the reservoir will inevitably block the pores Roar.

From the core plugging experimental results, the permeability of the reservoir increased after the acid liquid. Also, from the previous acid viscosity change data, it is known that the acid liquid can have a good gel-breaking effect on the fracturing fluid. The change of rate and the acid solution corrosion experiment know that acid solution can not only act on fracturing fluid but also dissolve reservoir minerals. Through the simultaneous effects of these two aspects, it has a good effect on removing plugging and increasing production.

(3) Experimental results and analysis of acid cleaning for supporting cracks.

The experimental results indicate that (as shown in Fig. 23) the permeability of the supporting fractures has greatly improved after the polyhydroacid cleaning, and the permeability increase rate is 50.0-53.2\%. Thus, the fracture permeability recovery level is higher.

(4) Microscopic experimental results and analysis of core damage.

Figure 24 shows the infrared analysis spectrum of the experimental sample.
From the analysis of some characteristic peaks appearing in the spectrum, together with the properties of the fracturing fluid, we can deduce whether the fracturing fluid is adsorbed on the formation rocks, and whether the acid fluid has a destructive effect on the damage of the fracturing fluid through the following:

1. By comparing the original shape of the rock block with the infrared spectrum of the rock block after flowing through the fracturing fluid. The rock block after flowing through the fracturing fluid has two obvious wave crests at 2920.62 $\left(\mathrm{cm}^{-1}\right)$ and $2852.2\left(\mathrm{~cm}^{-1}\right)$. Using the method of infrared spectrum characteristic analysis, this phenomenon should be the asymmetric stretching vibration Vas and symmetric stretching vibration Vs of methyl or methylene in the polymer. It shows that after fracturing fluid, there is fracturing fluid to be adsorbed on the rock surface, indicating that the fracturing fluid damages the reservoir.

2. By comparing the infrared spectra of the original rock block with fracturing fluid and then hydrochloric acid, there is no peak or obvious peak at $2920.62\left(\mathrm{~cm}^{-1}\right)$ and $2852.2\left(\mathrm{~cm}^{-1}\right)$. The spectrum of the rock block in the figure is almost the same as the original spectrum, indi-
Fig. 23 The results of the cleaning test of polyhydroacids on supporting cracks

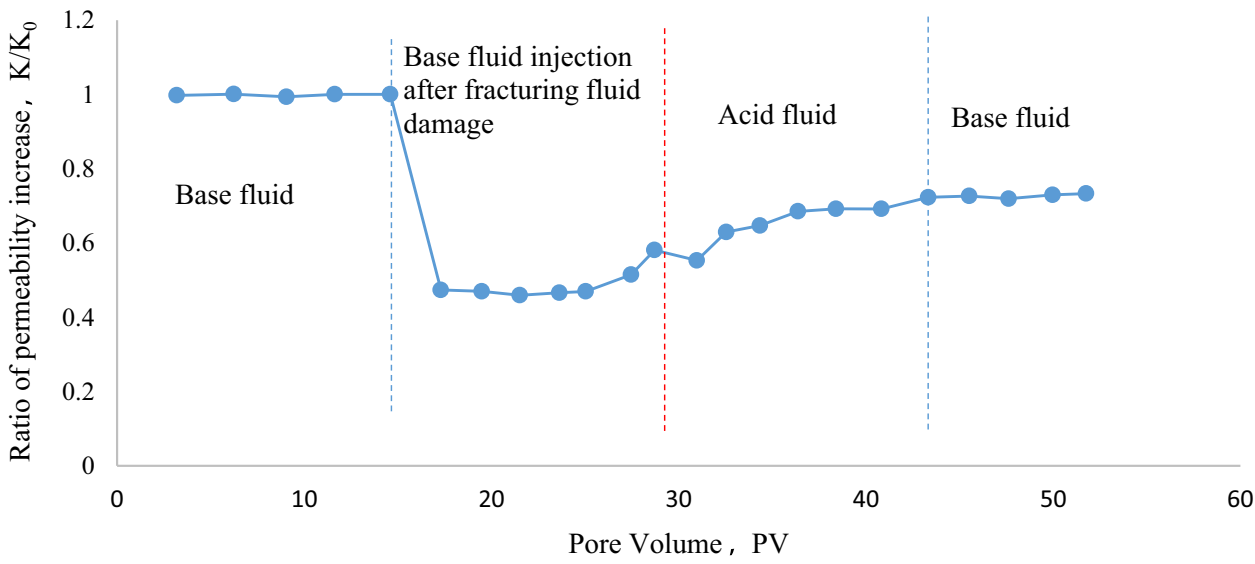




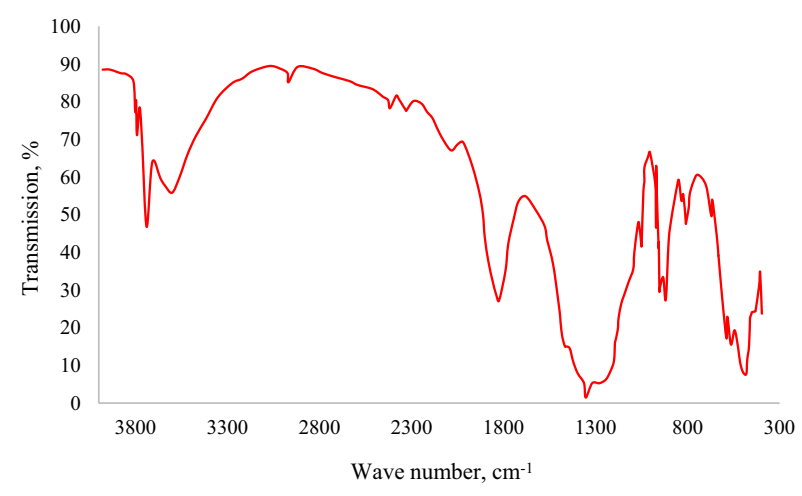

a Original image

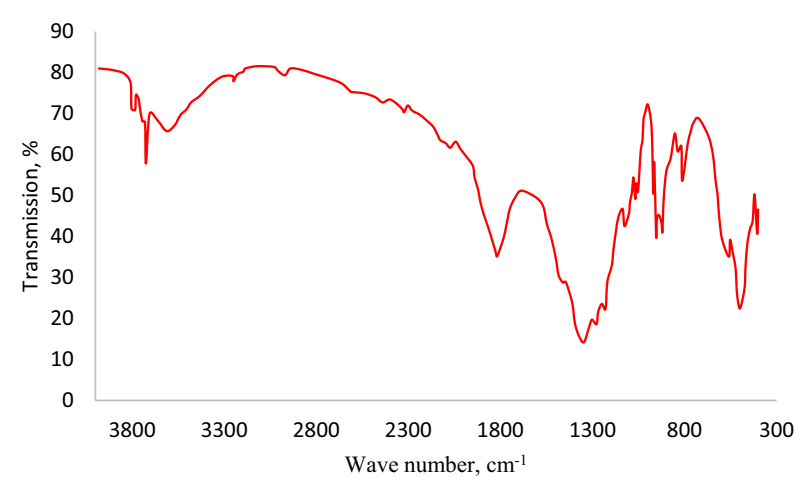

b Fracturing fluid passage diagram

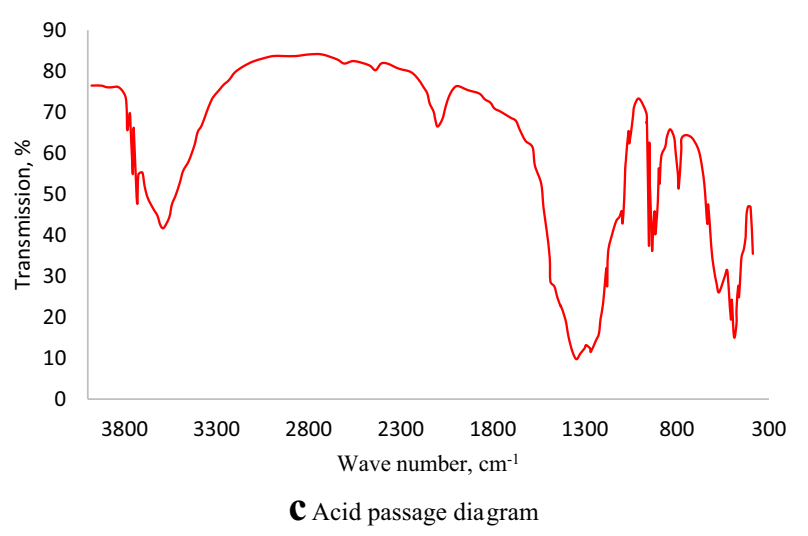

Fig. 24 Infrared analysis spectrum, a original image, b fracturing fluid passage diagram, $\mathbf{c}$ acid passage diagram

cating that the damage of the fracturing fluid is obviously relieved under the action of the acid, and the acid system can remove the damage of the fracturing fluid.

\section{Conclusion}

1. In the process of fracturing, hydroxypropyl guar gum will adhere to the surface of proppant, and the molecular chains of hydroxypropyl guar gum will be retained and molecular flocs will block the pore throat. Finally, the permeability decreased. The pre-acid fracturing technology combines acid treatment and propped fracturing technology, which can not only play the advantages of conventional fracturing to reform the reservoir, but also solve its existing problems to a large extent.

2. According to the characteristics of pre-acid fracturing and the performance requirements of pre-acid system, research on pre-acid formula is carried out. A new type of polyhydric acid system was developed, which was mainly composed of main agent SA601 and side agent SA701.

3. Through the evaluation of fracturing effect, we found that the new type of acid system can effectively reduce the fracture pressure of formation rock, promote the gel breaking of fracturing fluid, effectively inhibit the expansion of clay, and remove the damage of fracturing fluid.

Acknowledgment The work in this paper is supported by the grant from the National Natural Science Foundation of China (No. U1762107) and Science and technology program of Sichuan Province (No. 2019YJ0425).

Funding Funding was provided by National Natural Science Foundation of China (Grant No. U1762107).

Open Access This article is licensed under a Creative Commons Attribution 4.0 International License, which permits use, sharing, adaptation, distribution and reproduction in any medium or format, as long as you give appropriate credit to the original author(s) and the source, provide a link to the Creative Commons licence, and indicate if changes were made. The images or other third party material in this article are included in the article's Creative Commons licence, unless indicated otherwise in a credit line to the material. If material is not included in the article's Creative Commons licence and your intended use is not permitted by statutory regulation or exceeds the permitted use, you will need to obtain permission directly from the copyright holder. To view a copy of this licence, visit http://creativecommons.org/licenses/by/4.0/.

\section{References}

Cheng W, Ni J, Shen J, Wang Z (2018) Modeling of permeation and fracturing grouting in sand: laboratory investigations. J Test Eval 46(5):2067-2082. https://doi.org/10.1520/JTE20170170

Dang L, Zhou C, Huang M, Jiang D (2019) Simulation of effective fracture length of prepad acid fracturing considering multiple leak-off effect. Nat Gas Ind B 6(1):64-70. https://doi.org/10. 1016/j.ngib.2019.01.009

Dashti QM, Kabir MM, Raju V et al (2007) An integrated evaluation of successful acid fracturing treatment in a deep carbonate reservoir having high asphaltene content in burgan field, Kuwait. Int Pet Technol Conf. https://doi.org/10.2523/IPTC-11347-MS

Dunning JD, Miller ME (1984) Effects of Pore fluid chemistry on stable sliding of Berea sandstone. Pure Appl Geophys 122(85):447-462. https://doi.org/10.1007/BF00874611 
Fu L, Liao K, Ge J et al (2020) Study on the damage and control method of fracturing fluid to tight reservoir matrix. Coal Technol. https://doi.org/10.1016/j.jngse.2020.103464

Gdanski R, Fulton D (2006) Fracture face skin evolution during cleanup. Soc Pet Eng. https://doi.org/10.2118/101083-MS

Gensheng L, Sheng M, Shouceng T et al (2012) Multistage hydraulic jet acid fracturing technique for horizontal wells. Pet Explor Dev 39(01):107-112. https://doi.org/10.1016/S1876-3804(12)60021-4

Guo T, Li Y, Ding Y et al (2017) Evaluation of acid fracturing treatments in shale formation. Am Chem Soc 31(10):10479-10489. https://doi.org/10.1021/acs.energyfuels.7b01398

Hajiabadi SH, Bedrikovetsky P, Mahani H et al (2020) Effects of surface modified nanosilica on drilling fluid and formation damage. J Pet Sci Eng 194:107559. https://doi.org/10.1016/j.petrol.2020. 107559

Holditch SA (1979) Factors affecting water blocking and gas flow from hydraulically fractured gas wells. J Pet Technol. https://doi.org/ 10.2118/7561-PA

Hou B, Zhang R, Chen M et al (2019) Investigation on acid fracturing treatment in limestone formation based on true tri-axial experiment. Fuel 235(01):473-484. https://doi.org/10.1016/j.fuel.2018. 08.057

Huang Q, Liu S, Cheng W et al (2020) Fracture permeability damage and recovery behaviors with fracturing fluid treatment of coal: an experimental study. Adv Mech. https://doi.org/10.1016/j.fuel. 2020.118809

Jiang W, Ge H, Ning Z et al (2015) Research and application of the prepositioned acid fracturing technioue in Chang 8 acid-sensitive reservoir. Drill Prod Technol. https://doi.org/10.3969/J.ISSN. 1006-768X.2015.01.20

Jiawei K, Yan J, Kunpeng Z et al (2020) Experimental investigation on the characteristics of acid-etched fractures in acid fracturing by an improved true tri-axial equipment. J Pet Sci Eng. https://doi.org/ 10.1016/j.petrol.2019.106471

Jin X, Zhu D, Hill AD et al (2020) Effects of heterogeneity in mineralogy distribution on acid-fracturing efficiency. Soc Pet Eng 35(1):147-160. https://doi.org/10.2118/194377-PA

Kim CM, Losacano JA (1985) Fracture conductivity damage due to cross-linked gel residue and closure stress on propped 20/40 mesh sand. Soc Pet Eng. https://doi.org/10.2118/14436-MS

Krajcinovic D, Fonseka GU (1981) The continuous damage theory of brittle materials. J Appl Mech. https://doi.org/10.1115/1.3157739

Li T, Zhou D, Gu L et al (2012) Research and application of multicomponent Pad-acid fracturing technology in low permeability oilfield. Petrochem Ind Appl. https://doi.org/10.3969/j.issn.16735285.2012.10.013
Lufeng Z, Fujian Z, Shicheng Z et al (2019) Evaluation of permeability damage caused by drilling and fracturing fluids in tight low permeability sandstone reservoirs. J Petrol Sci Eng 175:1122-1135. https://doi.org/10.1016/j.petrol.2019.01.031

Peng HE, Hanmei LIU, Xiangdong LI et al (2013) Research and application of the pre-positioned acid fracturing technology in low permeability oilfield. J Yanan Univ (Nat Sci Ed). https://doi.org/ 10.3969/J.ISSN.1004-602X.2013.02.078

Prikryl R (2001) Some microstructural aspects of strength variation in rocks. Int J Rock Mech Min Sci 38(5):671-82. https://doi.org/10. 1016/S1365-1609(01)00031-4

Qi N, Chen G, Pan L et al (2020) Numerical simulation and analysis of fracture etching morphology during acid fracturing of dolomite reservoirs. Chem Eng Sci. https://doi.org/10.1016/j.ces.2020. 116028

Tan P, Jin Y, Hou B et al (2017) Experiments and analysis on hydraulic sand fracturing by an improved true tri-axial cell. J Pet Sci Eng 158:766-774. https://doi.org/10.1016/j.petrol.2017.09.004

Tang Q (2017) Application study of pre-acid fracturing technology for tight sandstone gas reservoir. Peirochem Ind Appl 36(05):38-42. https://doi.org/10.3969/j.issn.1673-5285.2017.05.009

Volk J, Gall BL (1983) Method for evaluation of formation damage due to fracturing fluids. Soc Pet Eng. https://doi.org/10.2118/ 11638-MS

Wang Y, Holditch SA (2008) Simulation of gel damage on fracture fluid cleanup and long-term recovery in tight gas reservoir. Soc Pet Eng. https://doi.org/10.2118/117444-MS

Wang J, Huang Y, Zhang Y et al (2020) Study of fracturing fluid on gel breaking performance and damage to fracture conductivity. J Pet Sci Eng 193:107443. https://doi.org/10.1016/j.petrol.2020.107443

Wu W, Sharma MM (2016) Acid fracturing in shales: effect of dilute acid on properties and pore structure of shale. Soc Pet Eng. https:// doi.org/10.2118/173390-PA

Xu B, Hill AD, Zhu D, Wang L (2011) Experimental evaluation of guar fracture fluid filter cake behavior. Soc Pet Eng 26(4):381-387. https://doi.org/10.2118/140686-PA

Zhang L, Tice M, Hascakir B (2010) A laboratory study of the impact of reinjecting flowback fluids on formation damage in the marcellus shale. Soc Pet Eng. https://doi.org/10.2118/195336-PA

Publisher's note Springer Nature remains neutral with regard to jurisdictional claims in published maps and institutional affiliations. 